\title{
MEGARA MOS: Where are my positioners and fibers pointing to?
}

Pérez-Calpena, Ana, Sánchez-Blanco Mancera, Ernesto, Gomez-Alvarez, Pedro, Martínez Delgado, Ismael, Castillo, África, et al.

Ana Pérez-Calpena, Ernesto Sánchez-Blanco Mancera, Pedro GomezAlvarez, Ismael Martínez Delgado, África Castillo, Armando Gil de Paz, Xabier Arrillaga, Marísa Luisa García Vargas, Jesús Gallego, Esperanza Carrasco Licea, Jorge Iglesias-Páramo, Raquel Cedazo, "MEGARA MOS: Where are my positioners and fibers pointing to?," Proc. SPIE 10706, Advances in Optical and Mechanical Technologies for Telescopes and Instrumentation III, 107062C (10 July 2018); doi: 10.1117/12.2311925

Event: SPIE Astronomical Telescopes + Instrumentation, 2018, Austin, Texas, United States 


\title{
MEGARA MOS: where are my positioners and fibers pointing to?
}

\author{
Ana Pérez-Calpena ${ }^{\mathrm{a}}$, Ernesto Sánchez-Blanco Mancera ${ }^{\mathrm{a}}$, Pedro Gomez-Alvarez ${ }^{\mathrm{a}}$, Ismael Martínez \\ Delgado $^{\mathrm{a}}$, África Castillo ${ }^{\mathrm{b}}$, Armando Gil de Paz ${ }^{\mathrm{b}}$, Xabier Arrillaga $^{\mathrm{c}}$, Marísa Luisa García Vargas ${ }^{\mathrm{a}}$, \\ Jesús Gallego $^{\mathrm{b}}$, Esperanza Carrasco Licea ${ }^{\mathrm{d}}$, Jorge Iglesias-Páramo ${ }^{\mathrm{e}}$, Raquel Cedazo ${ }^{\mathrm{f}} \&$ MEGARA \\ team \\ ${ }^{a}$ FRACTAL S.L.N.E., C/ Tulipán 2, Portal 13 1A, E-28231, Las Rozas de Madrid, Spain; \\ ${ }^{\mathrm{b}}$ GUAIX Group, Astrophysics Department, Universidad Complutense de Madrid, Av. Complutense \\ s/n, E-28040, Madrid, Spain; \\ ${ }^{\mathrm{c}}$ AVS, Pol. Ind. Sigma Xixilion Kalea 2, Bajo Pabellón 10, E-20870, Elgoibar, Gipuzkoa, Spain; \\ ${ }^{\mathrm{d}}$ Instituto Nacional de Astrofísica, Óptica y Electrónica, Luis Enrique Erro \#1, Tonanzintla, Puebla, \\ 72840, Mexico; \\ ${ }^{\text {e }}$ Instituto de Astrofísica de Andalucía, Glorieta de Astronomía s/n, E-18008, Granada, Spain; \\ ${ }^{\mathrm{f}}$ Facultad de Informática, Universidad Politécnica de Madrid, Campus de Montegancedo, E-28660, \\ Boadilla del Monte, Madrid, Spain;
}

\begin{abstract}
MEGARA is an integral-field and multi-object medium-resolution spectrograph for the GTC $10.4 \mathrm{~m}$ telescope, which was commissioned on June - August 2017. MEGARA offers two observing modes, the LCB mode, a large central IFU; and a Multi-Object Spectrograph (MOS) mode, composed by 92 robotic positioners carrying 7 -fiber minibundles each. This paper presents the models and measurements developed for the alignment between the image of the telescope pupil and the $100-\mu \mathrm{m}$ fiber cores during the integration and verification at the laboratory. On the one hand, the error in the positioner-minibundles assembly was optimized with the aim of achieving a fiber-to-fiber flux homogeneity better than $10 \%$. On the other hand, the positioner pointing was characterized in order to achieve a pointing precision of $1 / 5$ of the spaxel size (which has been designed to be $0.62 \mathrm{arcsec}$ ). The on-sky measurements obtained during the commissioning to verify our laboratory results are also presented.
\end{abstract}

Keywords: MEGARA, GTC, Multi-Object Spectrograph (MOS), optical fibers, fiber bundles, robotic positioners, tilt, pointing, characterization, verification

\section{INTRODUCTION}

MEGARA (Multi Espectrógrafo en GTC de Alta Resolución para Astronomía) is an optical fiber fed spectrograph [2]. The MEGARA focal plane subsystems are located at the Folded Cassegrain F (FC-F) focal station of the Spanish $10.4 \mathrm{~m}$ telescope GTC. Fiber bundles conduct the light from the FC-F to the spectrograph, hosted at the Nasmyth A platform (see Figure 1).

The instrument offers two observing modes: the one with the Integral Field Unit (IFU), named the Large Compact Bundle (LCB) mode, and the Multi-Object Spectrograph (MOS). Both modes are fed by $100 \mu \mathrm{m}$-core fibers. The IFU provides a field of view (FOV) of $12.5 \times 11.3 \mathrm{arcsec}^{2}$, while the MOS can place up to 92 mini-bundles with 7 fibers each covering a sky area of $3.5 \times 3.5 \mathrm{arcmin}^{2}$. Eight additional 7-fibers minibundles located at the edge of the $3.5 \times 3.5$ $\operatorname{arcmin}^{2}$ FOV (shown in orange at Figure 2) provide sky subtraction for the LCB mode. The science light comes into the spectrograph by means of a $44.5 \mathrm{~m}$ optical fiber link, which is routed through the FC-F rotator cable wrap, the elevation ring, the Nasmyth A elevation cable wrap and finally under the Nasmyth platform to reach the spectrograph entrance position (see Figure 1). Additional details about MEGARA fiber link design are provided at [6]. 


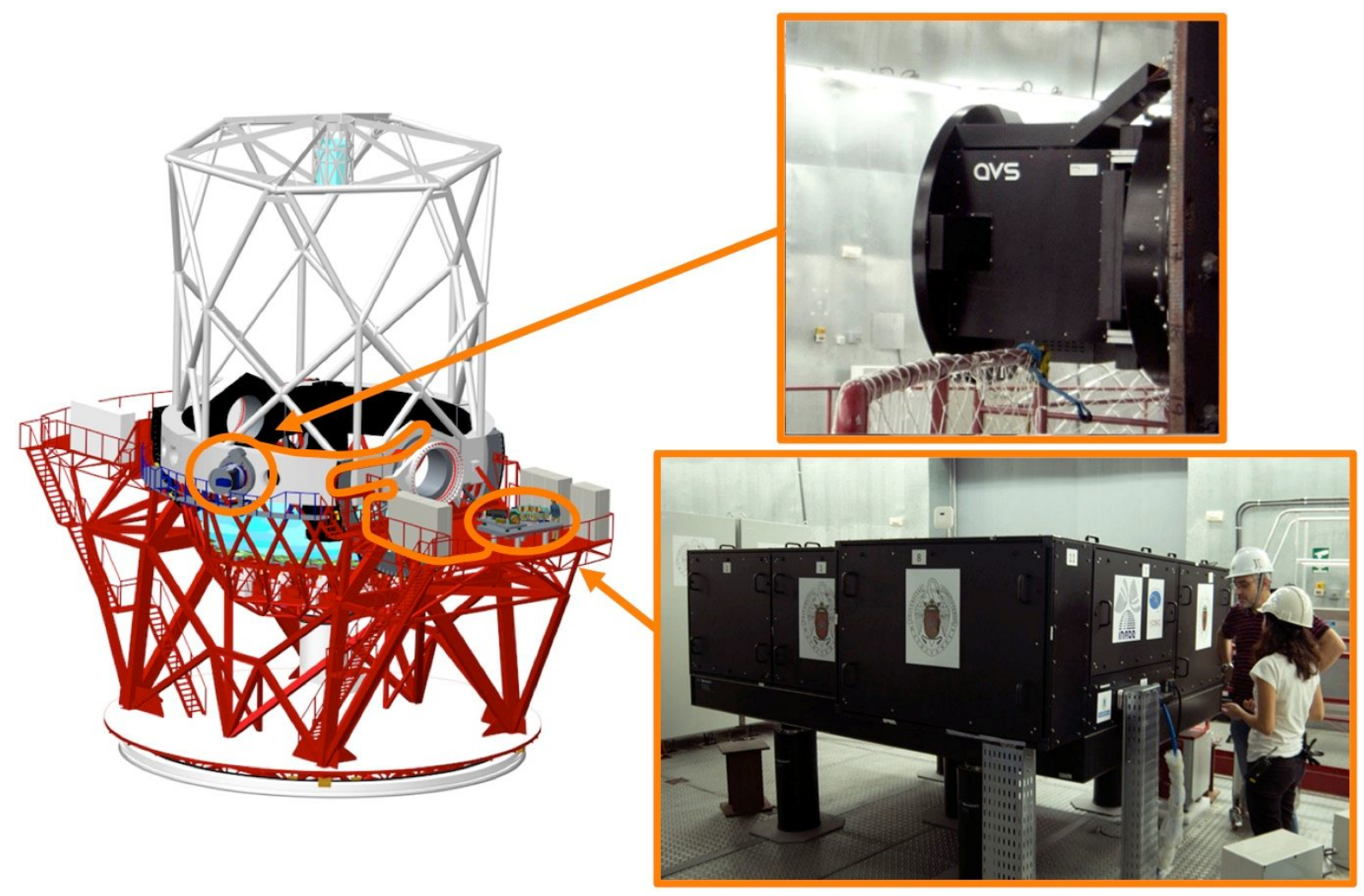

Figure 1: Left. A GTC 3D view showing MEGARA Folded Cassegrain subsystems integrated at FC-F focal station and MEGARA spectrograph at the Nasmyth-A platform. Right, a real view of MEGARA subsystems already integrated at the GTC.

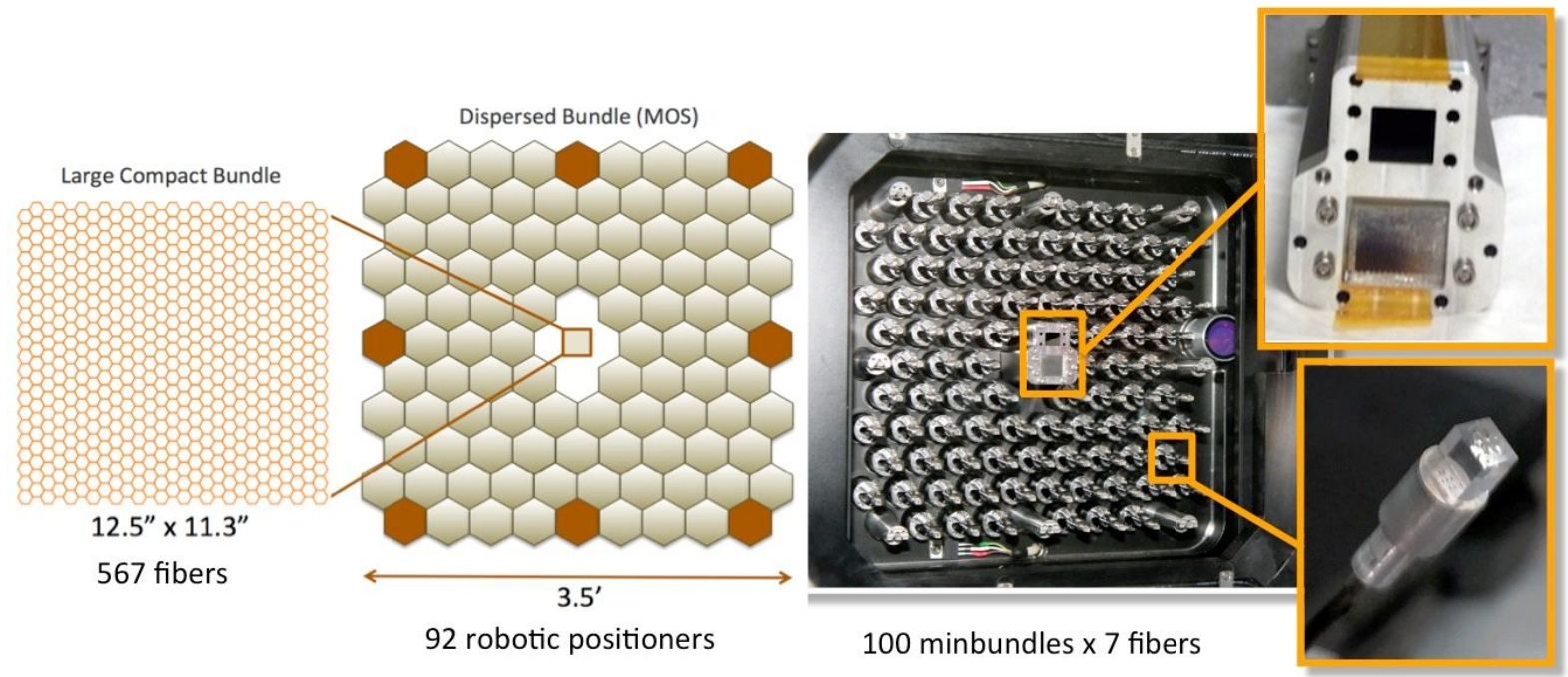

Figure 2: Left: MEGARA FOV layout. Right: View of the proper MEGARA focal plane and the details of the LCB and minibundles microlens arrays.

At the focal plane entrance, LCB and MOS fibers are telecentrically illuminated thanks to a field lens (Figure 3). Microlens arrays are coupled to the bundles to convert the telescope focal ratio F17 to F3 (Figure 2) for an optimum fitting to the telescope while keeping a minimum Focal-Ratio Degradation (FRD). Subsequently, each fiber bundle is placed at the spectrograph focal plane forming a smoothly curved pseudo-slit with $1075 \mathrm{~mm}$ radius of curvature and $110 \mathrm{~mm}$ length. 


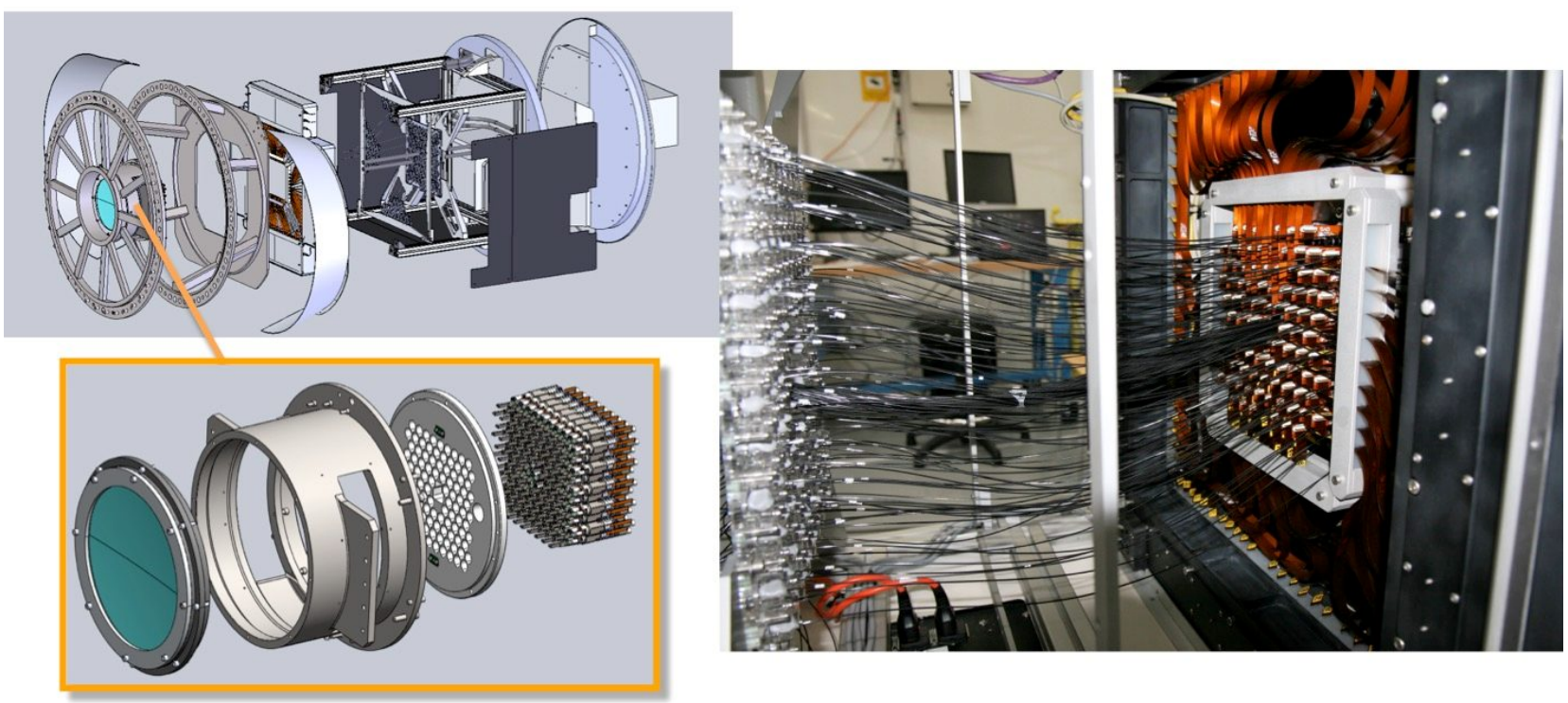

Figure 3: Left: Section of the Folded Cassegrain subsystems assembly and a exploded view of the optical components. Right: View of the minibundles arrangement inside the Fiber MOS assembly.

MEGARA main characteristics are summarized in Table 1.

Table 1: Main characteristics of MEGARA LCB IFU and MOS modes

\begin{tabular}{|c|c|c|c|}
\hline \multicolumn{2}{|c|}{ PARAMETER } & \multicolumn{2}{|c|}{ REQUIREMENTS } \\
\hline \multicolumn{2}{|c|}{ Telescope } & \multicolumn{2}{|c|}{ GTC } \\
\hline \multicolumn{2}{|c|}{ Plate Scale } & \multicolumn{2}{|c|}{$0.82 \mathrm{~mm} \mathrm{arcsec}^{-1}$} \\
\hline \multicolumn{2}{|c|}{ Field Lens } & \multicolumn{2}{|c|}{$\mathrm{D}=260 \mathrm{~mm}$} \\
\hline \multicolumn{2}{|c|}{ Microlens } & \multicolumn{2}{|c|}{$\begin{array}{c}\text { Hexagonal Shape } \\
\text { F-ratio transformation from F17 to F3 }\end{array}$} \\
\hline \multirow{2}{*}{\multicolumn{2}{|c|}{ Fiber Unit }} & \multicolumn{2}{|c|}{$\begin{array}{l}\text { Holded at Folded Cassegrain focus } \\
\text { Field lens for telecentric illumination }\end{array}$} \\
\hline & & Large Compact Bundle (LCB) & MOS Bundle \\
\hline \multicolumn{2}{|c|}{ Fiber-core } & $100 \mu \mathrm{m}$ & $100 \mu \mathrm{m}$ \\
\hline \multicolumn{2}{|c|}{ No. of fibers } & 623 & 644 \\
\hline \multicolumn{2}{|c|}{ Spaxel } & $0.62^{\prime \prime}$ & $0.62^{\prime \prime}$ \\
\hline \multicolumn{2}{|c|}{ FOV } & $12.5^{\prime \prime} \times 11.3^{\prime \prime}$ & $3.5^{\prime} \times 3.5^{\prime}$ \\
\hline \multicolumn{2}{|c|}{$\Delta \lambda\left(\mathbf{E E D}_{\mathbf{8 0}}\right)$} & 4.0 pix & 4.0 pix \\
\hline \multicolumn{2}{|c|}{$\Delta \lambda($ FWHM) } & 3.6 pix & 3.6 pix \\
\hline \multirow{3}{*}{$\mathbf{R}$} & LR & 6000 & 6000 \\
\hline & MR & 12000 & 12000 \\
\hline & HR & 18700 & 18700 \\
\hline \multicolumn{2}{|c|}{ Pseudo-slit } & \multicolumn{2}{|c|}{$110 \mathrm{~mm}$ long \& $\mathrm{ROC}=1075 \mathrm{~mm}$} \\
\hline \multicolumn{2}{|c|}{ F-ratios } & \multicolumn{2}{|c|}{ Collimator F3 to camera F1.5 } \\
\hline \multicolumn{2}{|c|}{ Pupils size } & \multicolumn{2}{|c|}{$160 \mathrm{~mm}$} \\
\hline \multicolumn{2}{|c|}{ Gratings } & \multicolumn{2}{|c|}{ VPHs, $240 \mathrm{~mm} \times 190 \mathrm{~mm}(\mathrm{MR}, \mathrm{HR}), 220 \mathrm{~mm} \times 180 \mathrm{~mm}(\mathrm{LR})$, selectable } \\
\hline \multicolumn{2}{|c|}{ Wavelength coverage } & \multicolumn{2}{|c|}{$3650 \AA-9800 \AA$} \\
\hline \multicolumn{2}{|c|}{ Detector } & \multicolumn{2}{|c|}{ E2V $1 \times 4 \mathrm{k} \times 4 \mathrm{k}, 15 \mu \mathrm{m}$ pixel size, AR coated } \\
\hline
\end{tabular}


The spectrograph is an all refractive system using a set of 18 interchangeable Volume Phase Hologram (VPHs) gratings. A wheel with two mechanisms (one to select the VPH and a second one to position the selected VPH in the pupil area in the collimated beam) handles the gratings to configure the selected set-up. The VPH set offers three spectral resolution modes: Low (LR), Medium (MR) and High (HR) resolution, which provides resolving power, $\mathrm{R}_{\mathrm{FWHM}}$, of 6000, 12000 and 18700 for LR, MR and HR respectively. LR and MR covers all the visible spectral range from $3650 \AA$ to $9800 \AA$, while the two HR units are centered at $6500 \AA$ and $8600 \AA$ respectively.

MEGARA was developed under contract with GRANTECAN S.A. Universidad Complutense de Madrid (UCM) is the leader of MEGARA Consortium, which is participated by Instituto Nacional de Astrofísica, Óptica y Electrónica (INAOE) in Mexico, the Instituto de Astrofísica de Andalucía (IAA-CSIC) and the Universidad Politécnica de Madrid (UPM) in Spain. The MEGARA Science Team includes a line-up expert researchers encompassing different astronomical areas from exoplanets and cool stars to cosmology, going through massive stars, planetary nebula, nearby and distant galaxies, coming from all the MEGARA consortium institutions and other Spanish and Mexican research centers together the University of Florida (UF).

MEGARA carried out the commissioning [1] between May and August 2017 (4 DayTime weeks 40 hrs each plus 3 NightTime runs 10 nights each). MEGARA has been offered to the GTC user community to start operations in July 2018.

\section{MEGARA MOS}

The MEGARA MOS mode is constituted by 92 robotic positioners carrying out a mini-bundle of 7 fibers coupled to a hexagonal pattern micro-lens array to convert the GTC F17 focal ratio to F3 to minimize FRD and to fit the entrance $\mathrm{f} / \mathrm{ratio}$ at the collimator. The spaxel size of each fiber is $0.62 \mathrm{arcsec}$, so each mini-bundle FOV is $\sim 1.8$ arcsec diameter. Robotic positioners provide two rotations that allow pointing 7 fiber mini-bundles in circular areas of $23.21 \mathrm{~mm}$ diameter, which overlap between adjacent positioners (distance between positioner centers is $20.1 \mathrm{~mm}$ ). The total FOV covered by the 92 positioners is 3.5 arcmin x 3.5 arcmin.

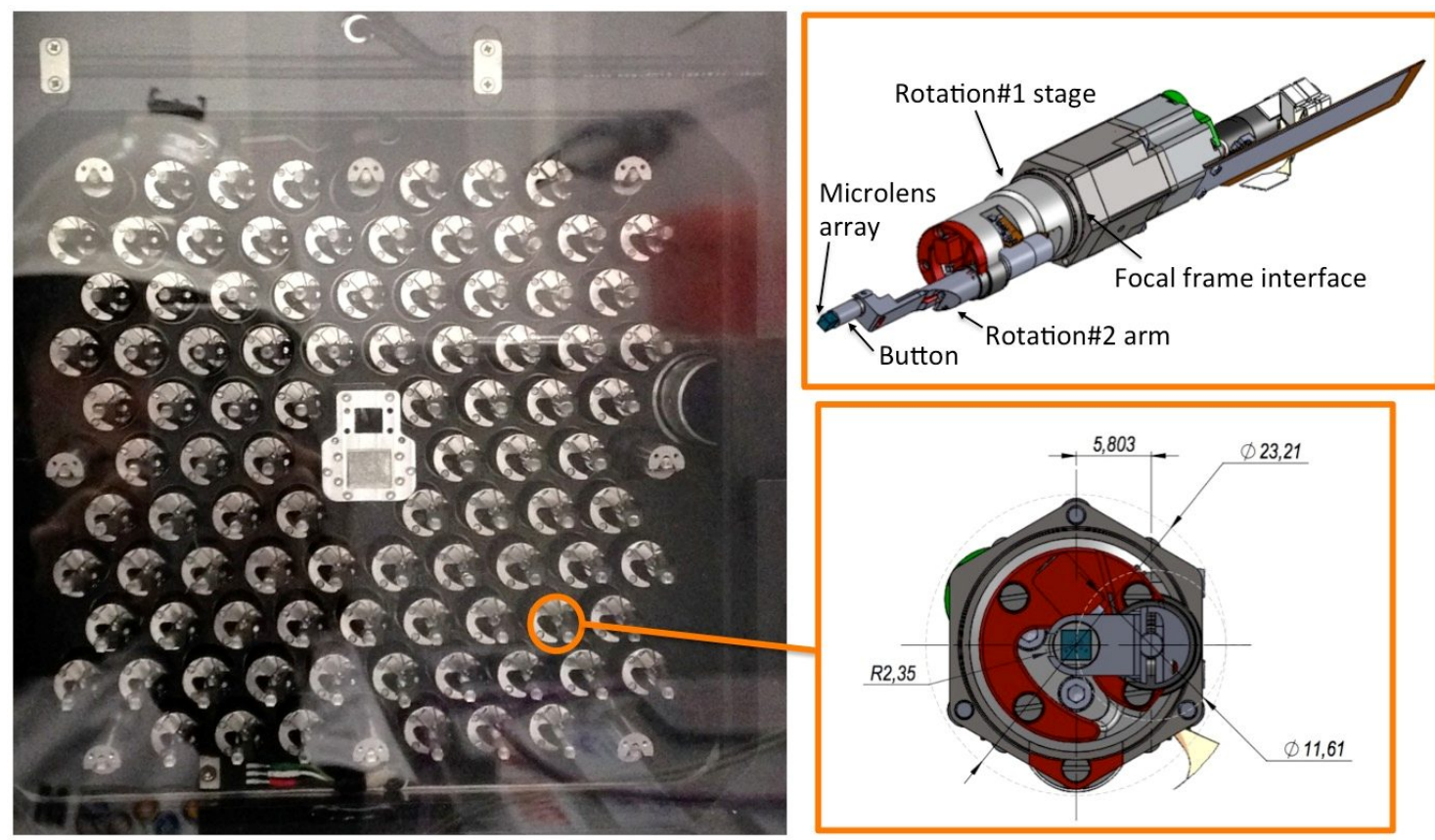

Figure 4: Left: As-built MEGARA focal plate, including the 92 positioners of the MOS mode as well as the LCB (the IFU, at the center) and the 8 LCB substraction sky-minibundles at the edges of the image. Right up: 3D model view of the robotic positioner. Right down: Fiber MOS drawing detail showing some relevant positioner dimensions. 
In order to provide the accuracy pointing and the throughput and flux uniformity required by the MEGARA Science Team, the MOS minibundles and positioners assembly had to fulfill the following two main requirements:

- Tilt: The MOS mini-bundle optical axis shall be better than 25 arcmin with respect to the telescope focal station optical axis in any pointing position in order to guarantee that the fiber is imaging at least the $90 \%$ of the GTC aperture (which is at the secondary mirror) and, therefore, to ensure fiber-to-fiber flux uniformity better than $10 \%$.

- Pointing: The positioner pointing accuracy shall be better than $1 / 5$ of the spaxel size in order to be able to ensure the centering of the target at the microlens.

These two high-level requirements were flown down to lower level requirements throughout system error budgets. Lower level requirements were formulated in terms of manufacturing and integration tolerances and component performance specifications, and then assigned to the corresponding subsystems and components to be verified at subsystem and components level before being finally verified at system level.

The manufacturing, integration and verification of the MOS mode was a complex process that required the intervention of different actors and the execution of several steps that were partially shared with the LCB manufacturing, integration and verification process and that can be summarized as follows:

- Robotic positioners and mechanical ends were designed and manufactured by the AVS company for both LCB and MOS modes. The mechanical ends, which are the parts where the fibers are integrated, include buttons (Figure 4) and pseudo-slit boxes. After dimensional verification, LCB frame, MOS buttons and pseudo-slit ends were sent to the fiber manufacturer, SEDI.

- Fibers were integrated at the LCB frame and positioner buttons (focal plane side) and at the pseudo-slit boxes (spectrograph side), glued and polished, by SEDI, who carried out the quality control of the bundles to verify fiber transmission. Then, both LCB and MOS fiber bundles were sent to the Laboratory of Advanced Instrumentation (LICA) at the Universidad Complutense de Madrid, where MEGARA system integration and verification was carried out.

- The gluing of the microlens arrays with the LCB and MOS fiber mini-bundles had been performed at LICA by FRACTAL S.L.N.E. The gluing task included the precise alignment between fibers and microlens plus the tilt verification of the complete assembly [5]. Once this task was completed, the LCB fiber link was ready to be integrated at the focal plane while the MOS fiber mini-bundles were ready to be integrated with the corresponding robotic positioners.

- The Folded Cassegrain assembly mechanical frame, which includes all mechanical parts for supporting optics and electronic components at the focal plane and providing fiber arrangement, was designed and manufactured by AVS. This frame was sent to LICA together with the first batch of positioners verified at AVS. Positioners' delivery continued during the positioner-minibundle integration process as soon as they were verified at AVS.

- The gluing of the positioner buttons (integrated to the fiber minibundles and microlens arrays) to the robotic positioners was performed by AVS at LICA. The optical verification of this assembly alignment and the dynamical tilt measurements was performed in parallel to the gluing tasks by FRACTAL.

- The LCB fiber link and the MOS minibundles (integrated at the robotic positioners) were routed through the Folded Cassegrain assembly structure and attached to the focal frame by AVS and FRACTAL.

- Finally, system tests of the LCB and MOS alignment and positioners pointing were also carried out at LICA, and their results were fully documented, before the delivery of MEGARA to GTC [3].

This step-by-step verification process allowed detecting any non-conformance and taking early corrective actions, which was the case when AVS reported that the requirements for dynamic tilt and pointing accuracy could not be guaranteed for all positioners due to a limitation in the gluing process carried out by the rotation 2 gear-motor provider. The effect of this limitation was that the distances between centers of rotation 1 and rotation 2 and the distance between rotation 2 center and the button center could be out of the assigned tolerances generating larger dynamic tilt and pointing errors than the one that have been initially required.

In order to meet high-level requirements for alignment and positioning, a system level approach, involving other subsystems as compensators was successfully applied as explained in detail in the following sections. 


\section{MOS TILT VERIFICATION}

MOS minibundles optical axis tilt must be better than 25 arcmin to fulfill the science requirement on flux homogeneity:

The fiber-to-fiber variation shall be less than $10 \%$ in the fraction of the pupil imaged to ensure a proper relativeflux calibration and cosmetics.

To allocate this requirement to all possible contributors, a detailed error budget for flux homogeneity (see Detailed design column at Table 2) was prepared for deriving the manufacturing tolerances of the frames supporting the fibers, the microlens diameters and positions at the array, the external fiber diameters, the positioning accuracy of the robotic positioner and, the performance of the gluing system between fiber and microlens.

Table 2: MOS mode flux homogeneity error budget.

\begin{tabular}{|c|c|c|}
\hline ITEM & Detailed design (rms) & AIV phase (rms) \\
\hline MOS button microholes position & $\pm 5.0 \mu \mathrm{m}$ & \\
\hline MOS button microholes diameter & $\pm 3.5 \mu \mathrm{m}$ & \\
\hline Microlens position & $\pm 5.0 \mu \mathrm{m}$ & \\
\hline Microlens diameter & $\pm 2.0 \mu \mathrm{m}$ & \\
\hline Microlens - fiber assembling and gluing & $\pm 5.0 \mu \mathrm{m}$ & $\pm 8.5 \mu \mathrm{m}$ \\
\hline MOS button - positioner gluing & $\pm 1.5 \mu \mathrm{m}$ & $\pm 1.5 \mu \mathrm{m}$ \\
\hline Positioner - focal frame attachment & $\pm 1.0 \mu \mathrm{m}$ & $\pm 1.0 \mu \mathrm{m}$ \\
\hline Focal frame - rotator attachment & $\pm 1.0 \mu \mathrm{m}$ & $\pm 1.0 \mu \mathrm{m}$ \\
\hline Positioners dynamic errors & $\pm 4.7 \mu \mathrm{m}$ & $\pm 4.7 \mu \mathrm{m}$ \\
\hline TOTAL & $\pm 10.8 \mu \mathrm{m}$ & $\pm 9.9 \mu \mathrm{m}$ \\
\hline Requirement & \multicolumn{2}{|c|}{ $\pm 12 \mu \mathrm{m}$ (goal: $\pm 10 \mu \mathrm{m})$} \\
\hline
\end{tabular}

The dimensional verification reports of the MOS buttons confirmed that the microholes were positioned with a tolerance better than $\pm 5 \mu \mathrm{m}$ and that fiber could be centered with a tolerance better than $\pm 3.5 \mu \mathrm{m}$. Also, the verification report of the microlens confirmed that they were positioned with a tolerance better than $\pm 5 \mu \mathrm{m}$ and the diameter was specified with a tolerance better than $\pm 2 \mu \mathrm{m}$.

The tilt error of the assembled fibers and microlens was minimized during the gluing process and, then, after gluing, measured again with an independent verification set-up. This measurement (see AIV phase column at Table 2) included not only the error introduced by the gluing process but also previous contributors coming from microlens, buttons manufacturing and fibers alignment at the microholes. The average error for the $92 \mathrm{MOS}$ buttons was $\pm 5 \mu \mathrm{m}$, which corresponds to 9 arcmin tilt.

The button and positioners gluing target value was also achieved. An average error less than $\pm 1.5 \mu \mathrm{m}$ (which corresponds to 3 arcmin tilt) was measured during this process.

The dimensional verification of the positioners, focal frames and rotator adapter were also performed and the requested value confirmed.

As anticipated in Section 2, the problem to fulfill the total tilt appeared when it was identified that the dynamic tilt requested to be below $\pm 4.7 \mu \mathrm{m}$ ( 9 arcmin tilt) was not met by all positioners. Positioners cover their patrol area thanks to two rotations. Rotation $1\left(360^{\circ}\right)$ dynamic tilt was measured by AVS to be well below 1 arcmin, otherwise rotation 2 dynamic tilt did not fulfill, in average, the requirement. 
The mean square root approach applied to compute the total error worked for splitting tilt requirement between the different contributions. However, it was important to consider that the tilt errors of the fiber-microlens assembly and the dynamic error of the positioner were not independent. On the contrary, these errors could be combined to be subtracted. To achieve that, not only the magnitudes of the tilt errors must be known but also the error angles in a reference position of the positioner patrol plane. Having that information, positioner and fiber minibundles could be combined to optimize total tilt error and, therefore, fulfill the total requirement.

Section 3.1 explains the mathematical model that was applied to combine errors and optimize matching positioners and minibundles. Following sections provides measurements done for verification at LICA and, finally, at GTC.

\subsection{Model to combine optimally positioners and fiber minibundles tilt errors}

The total tilt error of the MOS minibundles is not constant as it depends on a dynamical contribution, which is the positioner pointing (i.e. at which rotation 1 and rotation 2 angles the positioner has been commanded). As shown in Table 2, the main error contributions are the microlens-fiber minibundle error and the positioner dynamical tilt error. The other contributions can be neglected for this analysis. Besides, as commented in the introduction of Section 3, the rotation 1 tilt error was measured to be well below 1 arcmin and can also be neglected. Therefore, the two errors driving the total tilt are the microlens-fiber minibundle tilt error and the positioner rotation 2 tilt error. These errors are not independent. They will combine with different amplitude depending of the rotation 2 angle as shown in Figure 5.

To understand how to combine these errors, it is necessary to explain first how the minibundle (already integrated at the button) is glued in the positioner arm. The fiber minibundle is routed through the positioner shaft and both are attached at the gluing tool against the references that are included to ensure that the center of the button is at the positioner center when rotation $1(\mathrm{R} 1)$ and rotation $2(\mathrm{R} 2)$ are both at $0^{\circ}$ and that the rotation 2 tilt error is cancelled at this position. Therefore, the tilt error $(\alpha)$ of the rotation 2 shall be 0 when rotation 2 is equal to $0^{\circ}$ but will be $2 \alpha$ when rotation 2 is equal to $180^{\circ}$ (Figure 6).
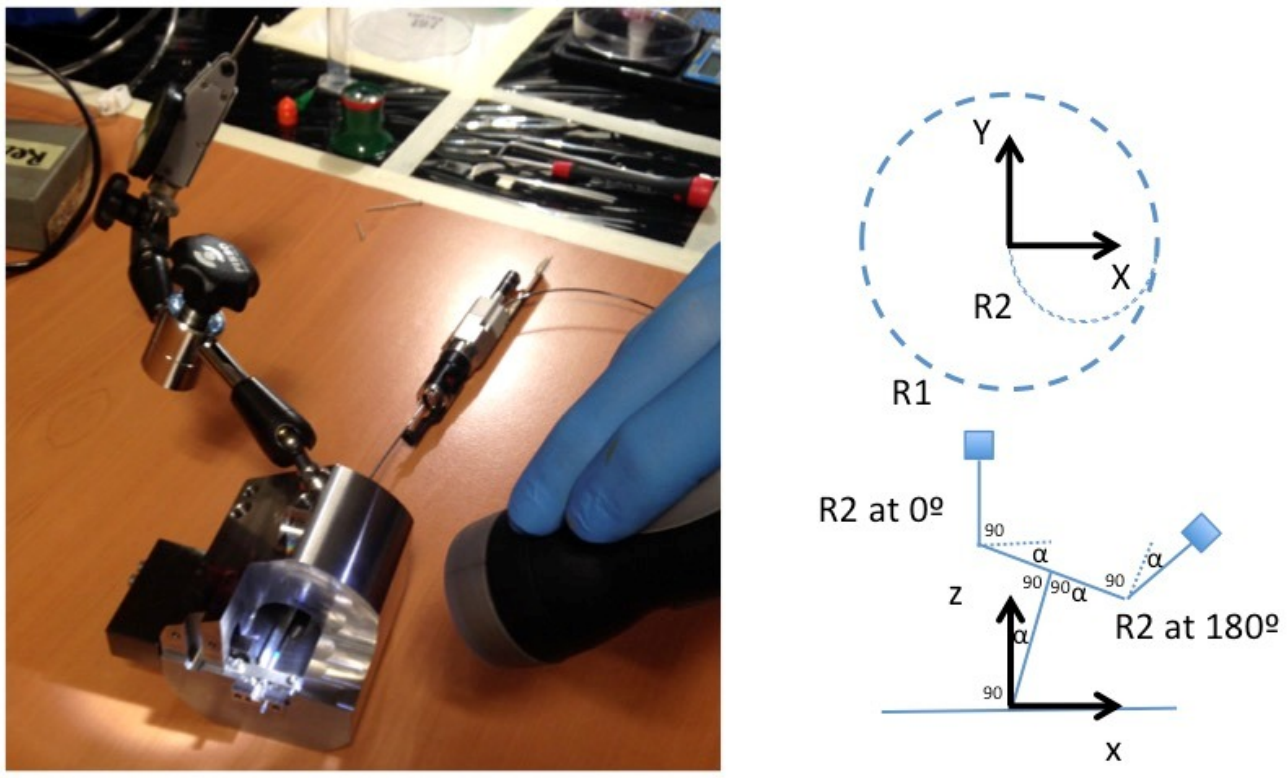

Figure 5: Left: Positioner-button gluing tool. Right: Schematic representation of the rotation 2 error at two positions $\left(0^{\circ}\right.$ and $\left.180^{\circ}\right)$.

Figure 6 shows the input data for this model: the microlens - fiber minibundle tilt error and the rotation 2 tilt error. 

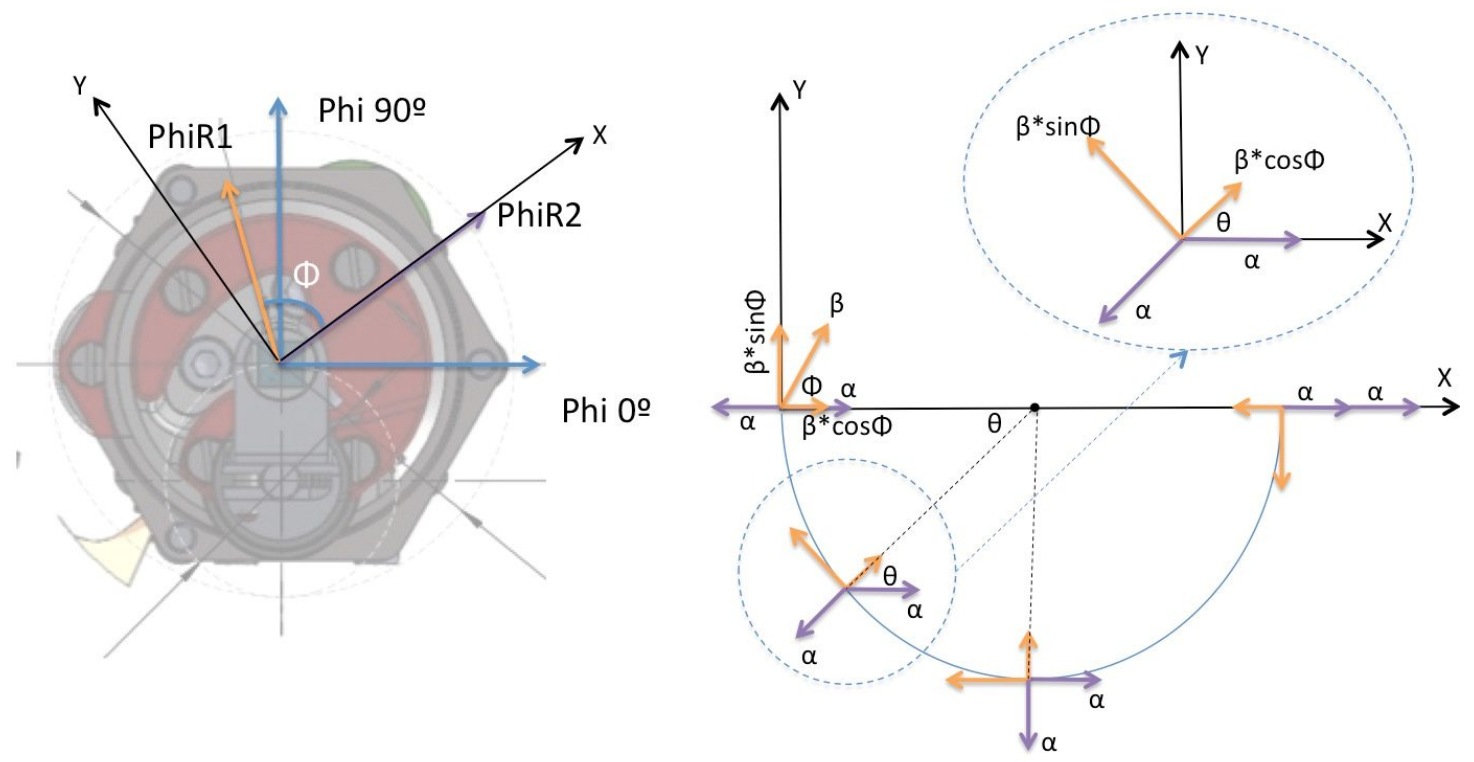

Figure 6: Left: Positioner axes at measuring orientation (blue) and positioner axes (black) for calculation of the combined error (positioner rotation 2 tilt error angle is used). Right: Combination of the tilt errors. Magenta arrows represent rotation 2 tilt error and orange arrows represents the microlens-fiber minibundle tilt errors. Both are decomposed in the x-y axes used in our calculations.

The first one was measured during the verification of the microlens and fiber minibundles gluing at LICA [5]. The microlens were always glued at the same orientation with respect to the button reference (reference marks included at the button and the microlens were used to keep always the same orientation between them). The button reference is then used at the microlens - fiber minibundle measurement verification set-up to obtain the magnitude $(\beta)$ and angle $\left(\Phi_{\mathrm{MB}}\right)$ of the minibundle-microlens assembly tilt error.

The magnitude $(\alpha)$ and angle $\left(\Phi_{\mathrm{P}}\right)$ of the positioner rotation 2 tilt error were measured at AVS.

Then, the following translations had to be applied to have all angles in the same reference system:

$$
\begin{aligned}
& \text { PhiR1 }=\Phi_{\mathrm{MB}}+\frac{\pi}{2} \\
& \text { PhiR2 }=\Phi_{\mathrm{P}}+\pi
\end{aligned}
$$

Then, the difference between these tilt error angles $(\Phi)$ is computed as:

$$
\Phi=\text { PhiR1 - PhiR2 }
$$

And the magnitude of the combined tilt error is computed as (being $\theta$ the angle of rotation 2):

Tilt $=\sqrt{(\beta(\cos \Phi \cos \theta-\sin \Phi \sin \theta)+\alpha(1-\cos \theta))^{2}+(\beta(\sin \Phi \cos \theta+\cos \Phi \sin \theta)-\alpha \operatorname{sen} \theta)^{2}}$

Therefore, having the magnitude and the angle of the rotation 2 tilt error of a given positioner, it could be combined with the different microlens-fiber minibundles data to choose the optimum fiber-minibundle.

\subsection{MOS minibundles individual tilt verification at laboratory}

After selecting and gluing each minibundle and positioner pair, the dynamical verification of the assemblies was carried out at the measuring set-up shown in Figure 7. This set-up was composed by a $120 \mathrm{~mm}$ focal lens and a detector with a pixel size of $5.5 \mu \mathrm{m}$. Fibers were back illumined so the pupil image of the fibers was projected on the detector. The positioner to be verified must be commanded to several positions at rotation 1 (R1) and rotation 2 (R2) to measure tilt errors and obtain model parameters. As example, Figure 8 shows results for a positioner-minibundle pair. 


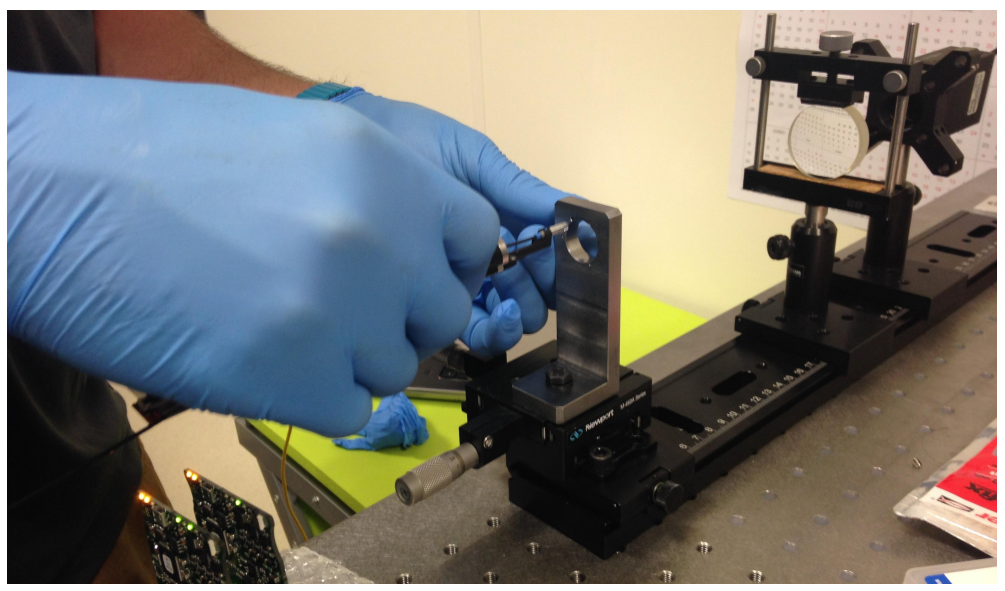

Figure 7: Introducing a positioner at the set-up for dynamical tilt verification.
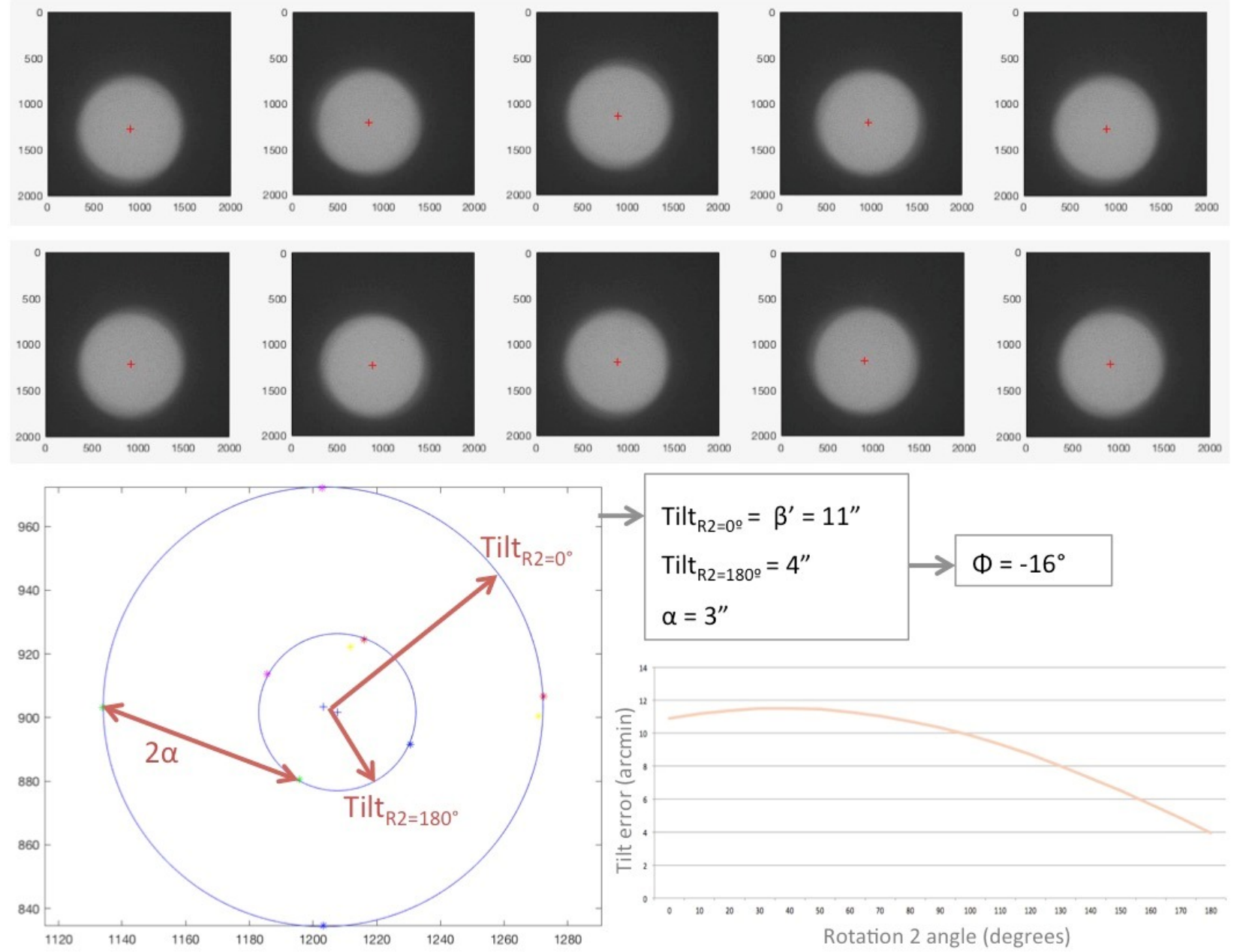

Figure 8: Up: Pupil images recorded at $\mathrm{R} 2=0^{\circ}$ and $\mathrm{R} 1=0^{\circ}, 90^{\circ}, 180^{\circ}, 270^{\circ}, 360^{\circ}$ (first row); and at $\mathrm{R} 2=180^{\circ}$ and $\mathrm{R} 1=0^{\circ}, 90^{\circ}, 180^{\circ}, 270^{\circ}$, $360^{\circ}$ (second row). Bottom left: Plot of the centroids identified at the previous images (units are in pixels), from this plot total tilt errors at $\mathrm{R} 2=0^{\circ}$ and $\mathrm{R} 2=180^{\circ}$ are measured as well as the rotation 2 tilt. Bottom right: Variation of the total tilt with rotation 2 angle as predicted by equation 4 with parameters $(\alpha, \beta$ ' and $\Phi)$. All figures correspond to minibundle $\# 19$. 
The centroids of the pupil images recorded in each position (see Figure 8 ) are used to measure the total tilt when rotation 2 is at $0^{\circ}$ and at $180^{\circ}$ as well as the rotation 2 tilt. Then, applying equation $4, \beta^{\prime}$ (which is the amplitude of the combined tilt error of the microlens-fiber minibundle plus button gluing) is obtained when $\theta=0^{\circ}$ while the angle difference $(\Phi)$ between PhiR1 and PhiR2 is calculated for $\theta=180^{\circ}$. It must be highlighted that $\beta$ and $\beta$ ' are normally very close as the button gluing contribution was in general lower than the microlens-fiber minibundle error and, therefore, the estimation done previously for matching positioner and minibundle worked always fine. After calculating these values, we obtain all parameters of the model to predict the total tilt at any angle of rotation 2.

As already explained in Section 3.1, it is relevant to mention that the total tilt error is considered constant in rotation 1. It means that the magnitude of the tilt error does not vary appreciably when a positioner is commanded at any angle inside the rotation 1 range while keeping a particular rotation 2 angle, so that only the angle of the tilt error varies. Therefore, the magnitude of the total tilt can be considered dependent only on rotation 2 angles and should remain constant for all rotation 1 range.

This individual verification was performed for each minibundle \& positioner pair. Figure 9 shows the tilt error for all 92 minibundles in the full rotation 2 range. All errors were below 25 arcmin and the average error was 12 arcmin.

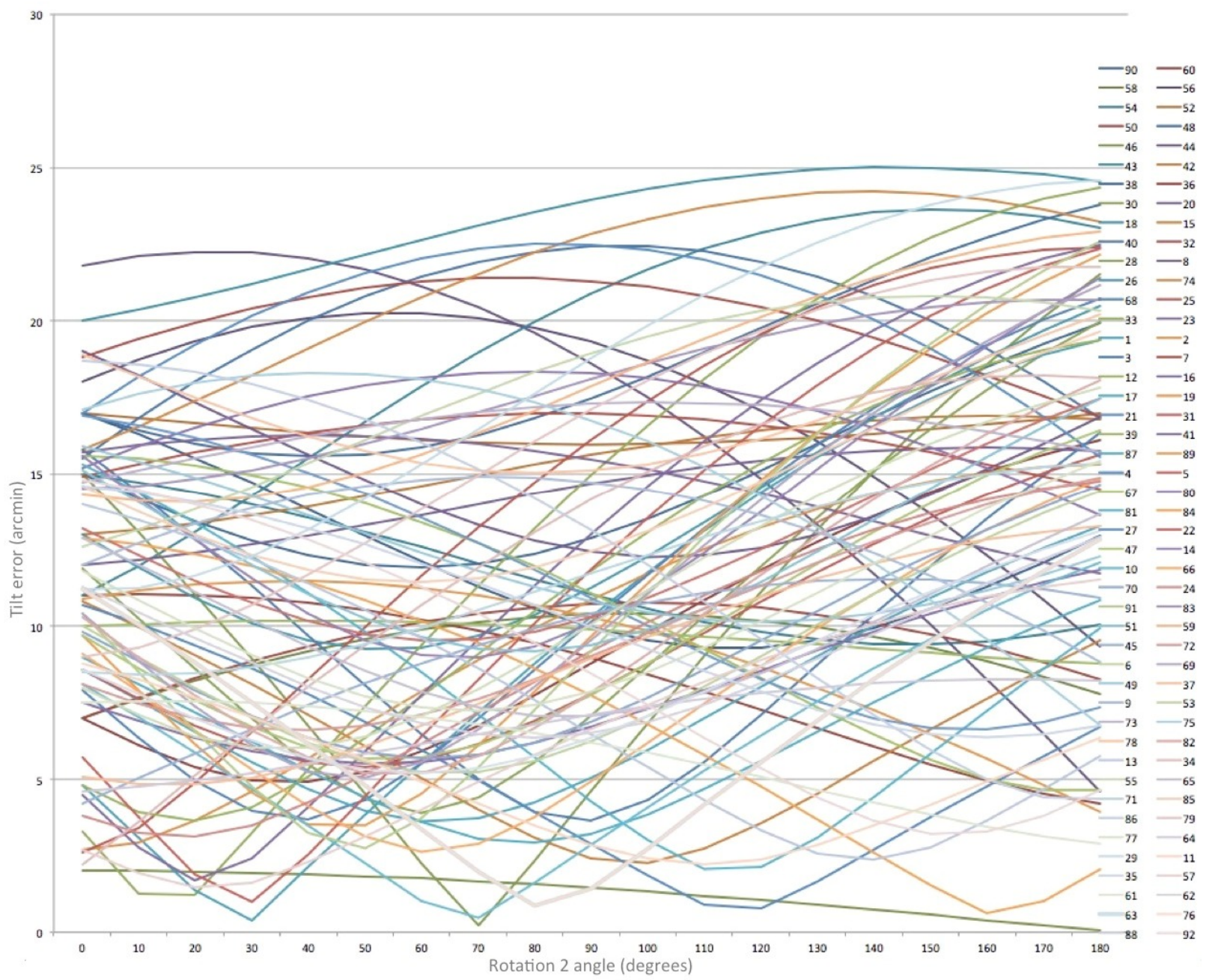

Figure 9: Tilt errors $(\operatorname{arcmin})$ for the whole range $\left(0-180^{\circ}\right)$ of the rotation 2. 


\subsection{MOS assembly tilt verification at laboratory}

After alignment verification of the individual MOS minibundles, MOS positioners were integrated together with the IFU at the focal frame.

A system test using a secondary mirror (M2) simulator was performed to confirm the assembly alignment, which, as explained in the introduction of Section 3, had been previously checked by dimensional verification of the mechanical parts.

The M2 simulator generated an F\#17 pupil image and was placed at $18 \mathrm{~m}$ from the MOS focal frame. The simulator was composed by 9 pairs of LEDs (see Figure 10) located at the center of the simulated mirror (LED 0), at 295 mm (LEDs 1 to 4 ) and at $478 \mathrm{~mm}$ (LEDs 5 to 8 ), which is the edge of the M2 pupil as seen by MEGARA.

The LEDs were illuminated pair by pair to characterize the pointing of the pupil in the fiber core. MEGARA CCD detector images were taken at each position with both modes (LCB and MOS), while positioners were commanded at different angles in order to verify that the pupil remained aligned with the fiber core for different positioner configurations. Then, images were processed by integrating the flux in two regions (bottom and up of the detector) for the LCB mode and in each minibundle (92 regions) for the MOS mode (see Figure 11).

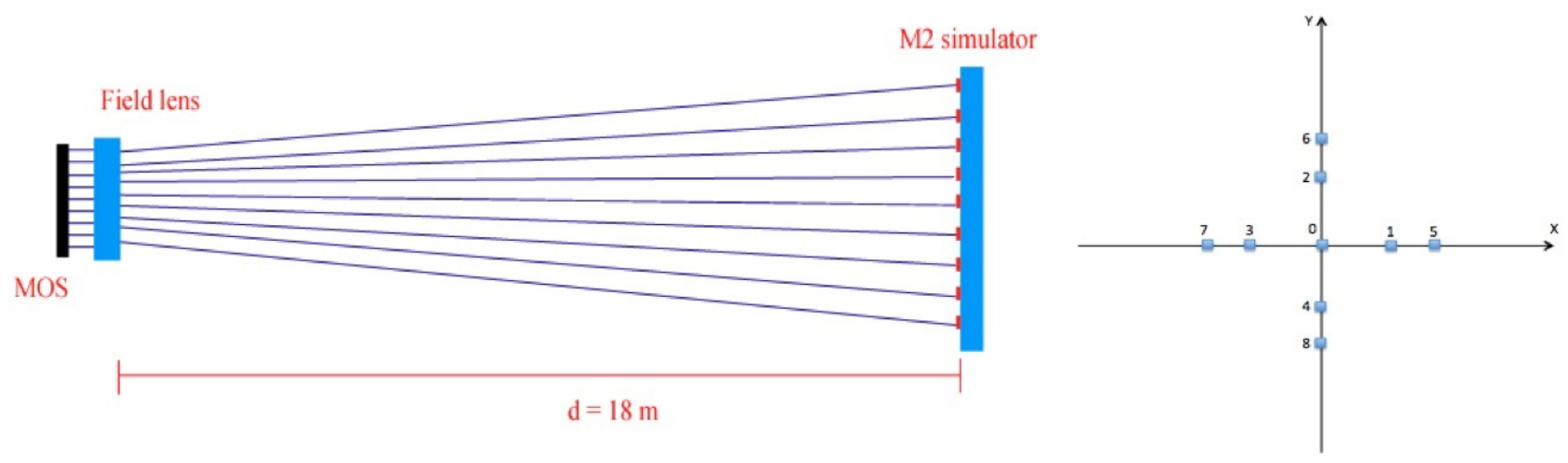

Figure 10: Left: Set-up for illuminating the complete MOS FOV with the M2 simulated mirror (figure is not scaled). Right: Layout of the M2 simulator mirror composed by 9 LEDs.
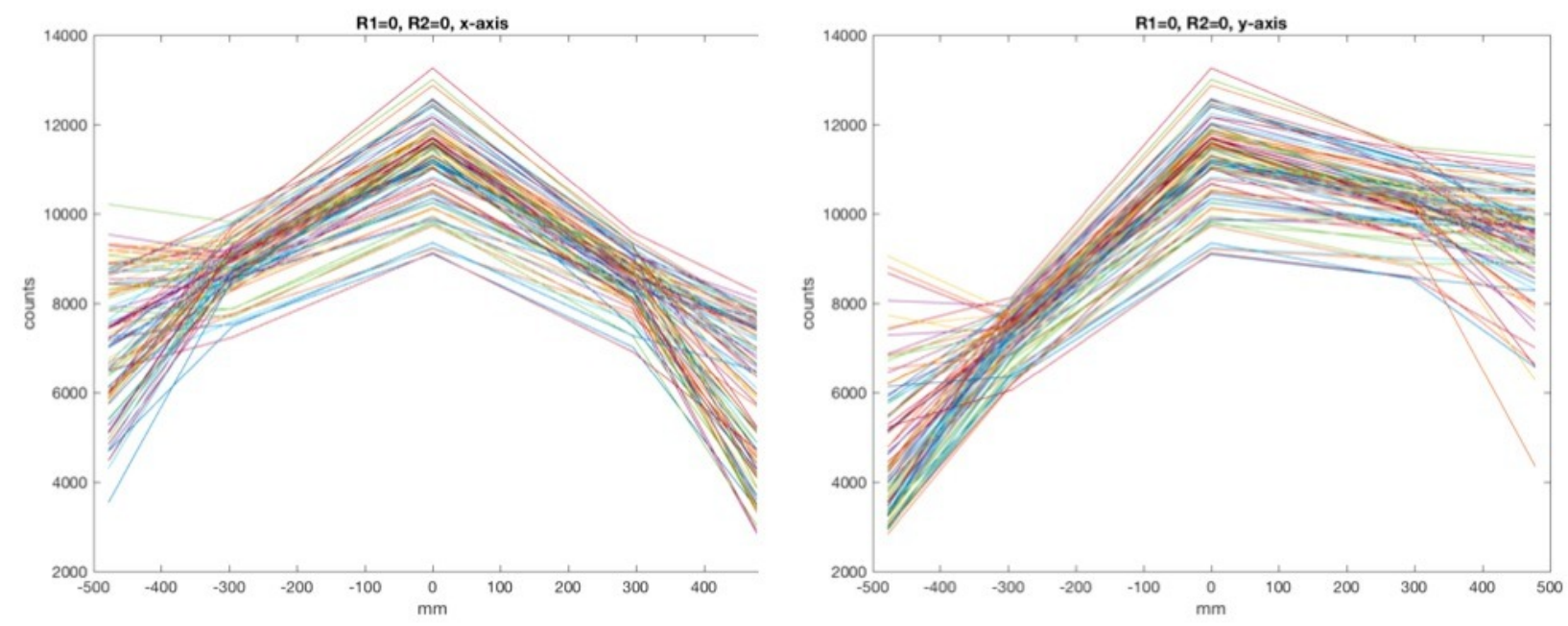

Figure 11: MOS flux measurements at the M2 simulator x-axis (left) and y-axis (right) at R1 $\left(0^{\circ}\right), \mathrm{R} 2\left(0^{\circ}\right)$ 
The integrated flux showed a good symmetry (flux level) in the x-axis but a worse symmetry in the $y$-axis, which was later assessed to be due to an on-axis misalignment of the M2 simulator itself. In addition, graphs showed that there is a flux variation registered at the different minibundles for a given LED, which could be due to differences in transmission of the fibers and/or the lack of homogeneity of the LEDs. Finally, graphs showed that the light from a particular LED was not fully entering or disappearing at a given minibundle in function of the minibundle tilt, as would have been expected. We understood this to be due, on the one hand, because LEDs were not providing a punctual image but a small spot and, on the other hand, because the light being propagated at the fibers depended on the angle of incidence (i.e., an angular scrambling could be expected for the light entering at the sides of each fiber).

Even taken these limitations into account, this test allowed us to analyze flux variation between minibundles at the different LEDs for several pointing positions and the conclusion was that the same flux distribution was found for the central LEDs (0) and for the LEDs 1 to 4 at intermediate positions, which indicated that there was no flux difference due to tilt up to these positions (as expected since these LEDs were located at 30' from the edge while the expectation for the tilt was to be always below $25^{\prime}$ ). We measured that the flux distribution varied for the outer LEDs (5 to 8 ), where the minibundles efficiency starts to be affected by the tilt depending on the tilt angle of each minibundle, following the same tendency measured at the individual positioners verification (as shown in Figure 9).

\subsection{MOS alignment verification at GTC}

Once at the GTC, and after a series of checks in the instrumentation room before installation, we integrated MEGARA at the GTC [3]. In particular, the Folded Cassegrain assembly (also named Fiber-MOS assembly) was integrated at the FC$\mathrm{F}$ focal station. Then, we verified the MOS alignment in two steps: first, the tilt of the MOS focal frame was measured by using a boresight telescope integrated at the MOS focal frame and, second, the dynamical tilt of the MOS minibundles was verified. Both tests were performed using dome lamps illumination.

\subsection{MOS focal frame alignment verification at GTC}

The alignment of the MEGARA MOS focal frame (which contains both the IFU and the MOS minibundles) with respect to the telescope axis was verified by using a boresight telescope that had been integrated at the MOS focal frame. Adjustment screws had been included at the MEGARA Folded-Cassegrain assembly to tilt and move in $\mathrm{z}$ axis the MOS frame, if needed.

The boresight telescope provided an image of M2, including its central hole (Figure 12, left). Keeping the FC-F rotator at $0^{\circ}$ position, the boresight telescope was rotated in $\mathrm{z}$ axis and images were taken in each angle (Figure 12, center). Then, the FC-F rotator was rotated to different angles and another set of images were taken (Figure 12, right).
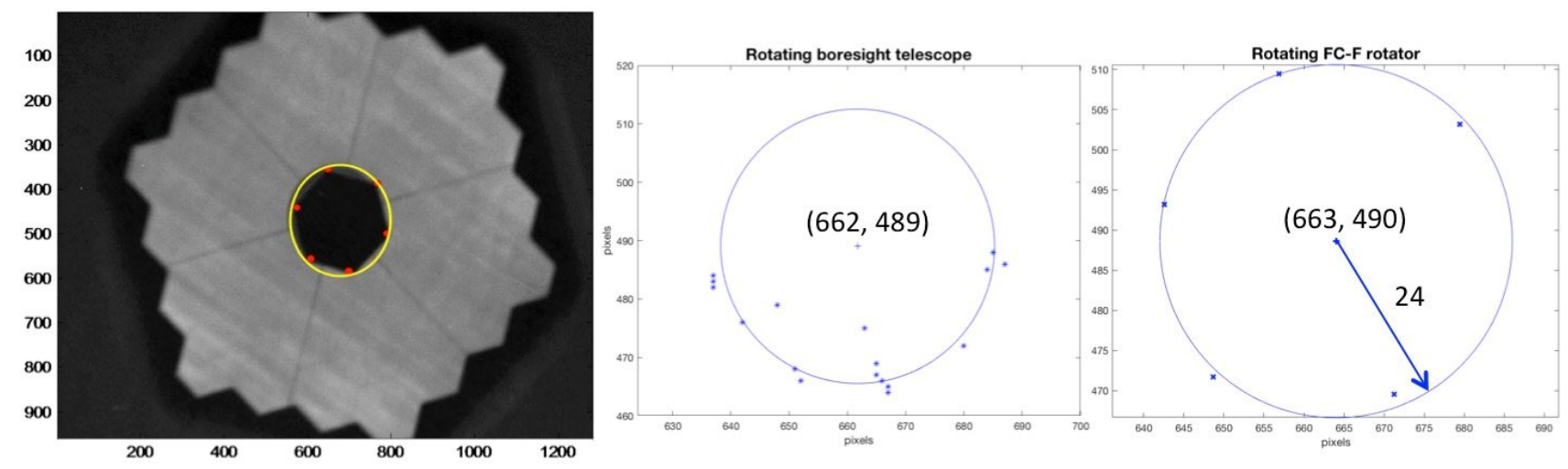

Figure 12: Left: MEGARA boresight telescope view of the GTC secondary (M2) mirror, which is telescope pupil. Center: M2 centers when boresight telescope was rotated. Right: M2 centers when FC-F rotator was rotated. 
The center of the boresight rotation was found at coordinates $(662,489)$ while the center of the rotator FC-F rotation was found at coordinates $(663,490)$ in units of pixels of the off-the-shelf boresight telescope camera. Considering that the boresight camera scale plate is $0.21 \mathrm{arcmin} / \mathrm{pixel}$, we concluded that the tilt between the FC assembly axis and the FC-F rotator axis was below 0.5 arcmin. Our requirement was to be below $0.03^{\circ}(1.8 \mathrm{arcmin})$ so no additional adjustment was needed.

We also found that the rotator axis described a 24 pixels radius circle. This meant that the FC-F rotator axis had a tilt with M2 - M3 axis (i.e., with the telescope optical axis at the FC-F focal station) of 5 arcmin. This was communicated to GTC staff, which then confirmed our measurements. This is relevant to be mentioned as it affected the dynamical tilt measurements performed afterwards and described in Section 3.6.

\subsection{MOS dynamical tilt verification at GTC}

To carry out the MOS dynamical tilt verification, MOS minibundles were illuminated through the telescope mirrors using the dome lamps. Several exposures were recorded with the positioners commanded at the following positions: (1) homing ( $\mathrm{R} 1=0^{\circ}, \mathrm{R} 2=0^{\circ}$, also called origin position); (2) positioner arm fully extended ( $\left.\mathrm{R} 1=0^{\circ}, \mathrm{R} 2=180^{\circ}\right)$ : (3) homing; (4) positioner arm fully extended and rotation 1 rotated $180^{\circ}\left(\mathrm{R} 1=180^{\circ}, \mathrm{R} 2=180^{\circ}\right)$ and (5) homing.
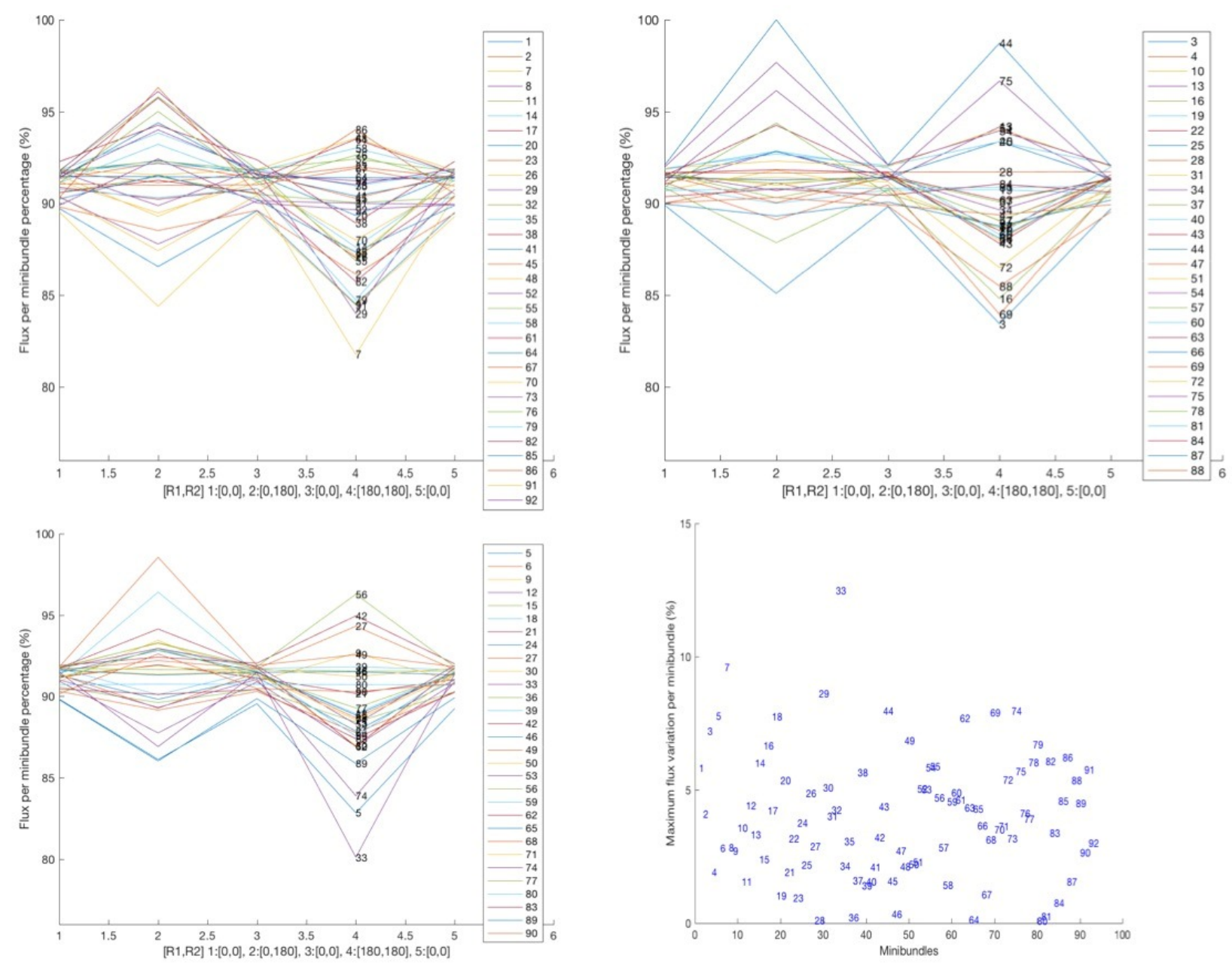

Figure 13: Up (left and right) and bottom (left): Percentage of flux per minibundle (with respect to the maximum measured flux) normalized to the flux of the static positioners $(36,28,64$ and 81$)$ during the tests, in the five positions indicated at the $x$ axis of each graph. Positioners were moved in 3 groups. Bottom (right): Maximum flux variation per minibundle. 
Movements of the positioners were performed in three groups to avoid moving adjacent positioners at the same time and, therefore, to prevent collisions. Positioner minibundles 36, 28, 64 and 81 were not moved and were used to normalize flux between positions to correct dome lamps variations (which were measured to be approximately $3 \%$ ).

Figure 13 shows percentages of the integrated flux per minibundle and per commanded position with respect to the maximum flux measured (graphs up left and right and down left). Bottom right panel of Figure 13 shows the maximum flux variation per minibundle computed as the percentage between the minimum and maximum flux integrated in each minibundle.

As explained in Section 3.2, the magnitude of the tilt is dependent on rotation 2 angle and remains constant for rotation 1 angle, so we expected that the flux variation between positions 2 and 4 (in both positions rotation 2 is at $180^{\circ}$, the positioner arm is fully extended, while rotation 1 is at two opposite angles, $0^{\circ}$ and $180^{\circ}$, in each position) should be symmetric. This was not happening for all minibundles (e.g. minibundles 7 and 33) and could be due to any of the following two reasons: (a) the rotation 1 contribution could be slightly larger than expected for some minibundles and (b) due to the 5 arcmin misalignment between the FC optical axis and the FC rotator axis which produces a lack of symmetry in the minibundle vision of M2 (GTC pupil). In summary, the misalignment of the optical axis will combine the minibundle dynamic tilt error, increasing or decreasing depending on the difference between the optical axis tilt error angle, which stays constant, and the angle that the minibundle tilt error would take at the different positions.

Even so, we could verify that the maximum variation of flux per MOS minibundle is less than $10 \%$ (with the only exception of minibundle 33 , what is around $12 \%$ ).

\section{MOS POINTING VERIFICATION}

The MOS pointing precision was required to be as follows:

MOS pointing precision shall be better than 1/5 of the spaxel size in order to be able to ensure the centering of the target at the microlens.

This means that the pointing precision must be better than 0.124 arcsec or $102 \mu \mathrm{m}$.

This requirement was applied to define the resolution of the motors and encoders for rotation 1 and 2, as well to specify the mechanical manufacturing tolerances for the positioner parts. Nevertheless, after identifying that the limitation in the gluing process of the rotation 2 gear-motor was preventing to achieve the required precision for rotation 2, the approach was to characterize each positioner parameters to take real geometry into account in the Fiber MOS software tool in order to be able to compensate the differences between a real positioner and the nominal one and to achieve the required pointing precision.

\subsection{Positioners characterization}

Positioner manufacturer (AVS) performed a dynamical characterization of each positioner geometry measuring, with a Zeiss O-Inspect 322 machine, the theoretical minibundle center of the positioner at several pointing positions with respect to a reference point in the test focal frame where the positioner was attached.

As shown in Figure 14, the 6 parameters that were required to characterize the positioners pointing were the $(\mathrm{x}, \mathrm{y})$ coordinates of P0, P1 and P3. This 6 parameters can also be obtained as: (1) and (2) as (x,y) coordinates of P0; (3) distance between R1 and R2 centers (P0 - P1); (4) distance between R2 center and minibundle center (P1 - P3); (5) angle between P0 - P1 axis and x-axis (OffsetR1) and (6) angle between P1 - P3 axis at homing position and P0 - P1 axis (OffsetR2).

These values are then used as configuration parameters in the Fiber MOS Assignment Tool (FMAT), the Fiber MOS Positioning Tool (FMPT) and the Fiber MOS Control Software (FMCS). The FMAT is the software tool used for selecting MOS targets on sky and assigning them to positioners [4]. The FMPT is the software tool used to calculate the trajectories of the positioners in order to reach the target positions and to provide the trajectories in motor steps per each rotation to the FMCS. Finally, FMCS is the MEGARA control system device in charge of executing positioner movements. Considering real geometry parameters at the software tool allowed fulfilling precision pointing requirement. 


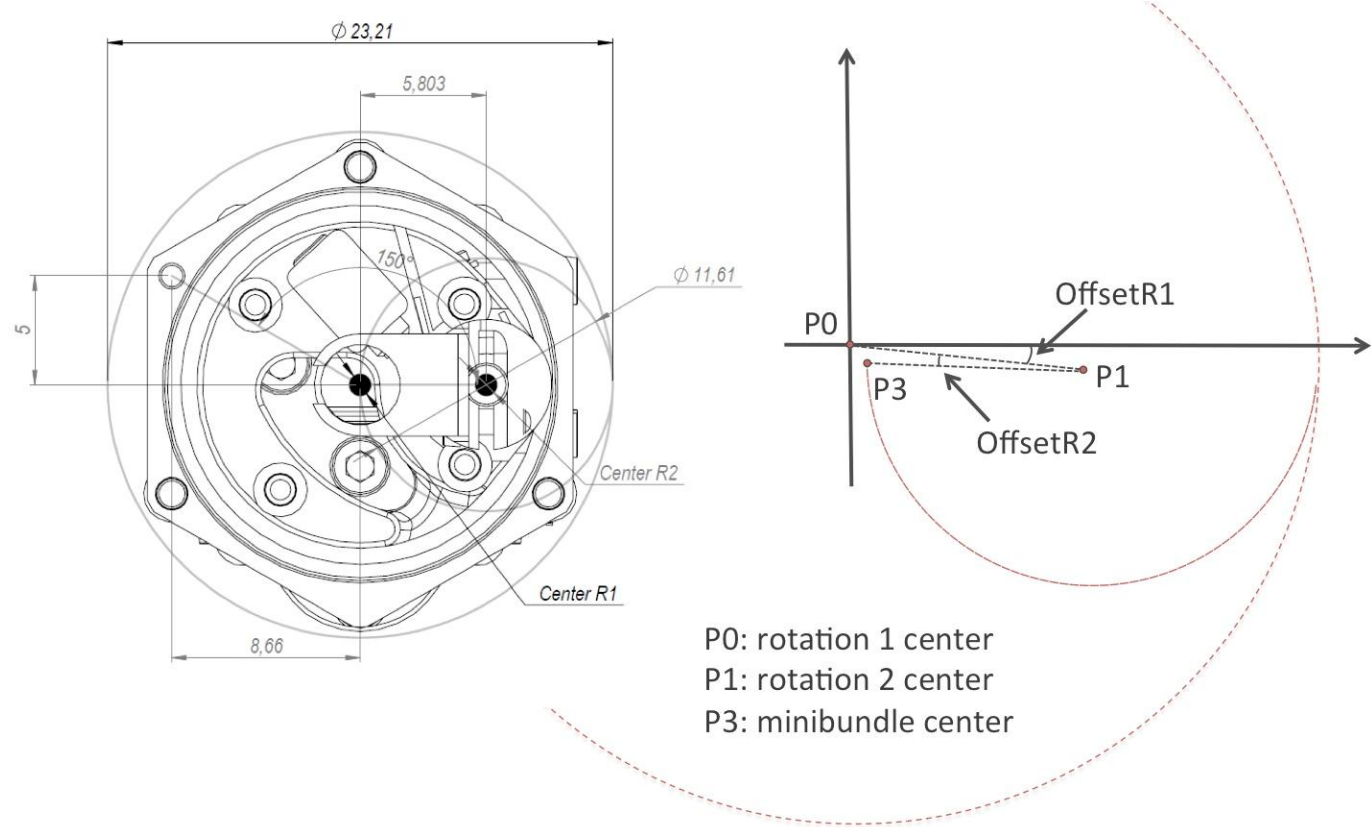

Figure 14: Left: Positioner nominal geometry. Right: Parameters to characterize a real positioner.

\subsection{Verification at laboratory of the MOS positioners' pointing parameters}

The approach to verify the MOS positioners' pointing parameters at laboratory included the following tasks: a) to take images of the back illuminated minibundles (with a uniform illumination through the MOS pseudo-slit) at different positions in rotation 1 and rotation 2 ; b) to identify centroids of the minibundles at those positions; and c) to determine from this information the 6 pointing parameters identified in Section 4.1.

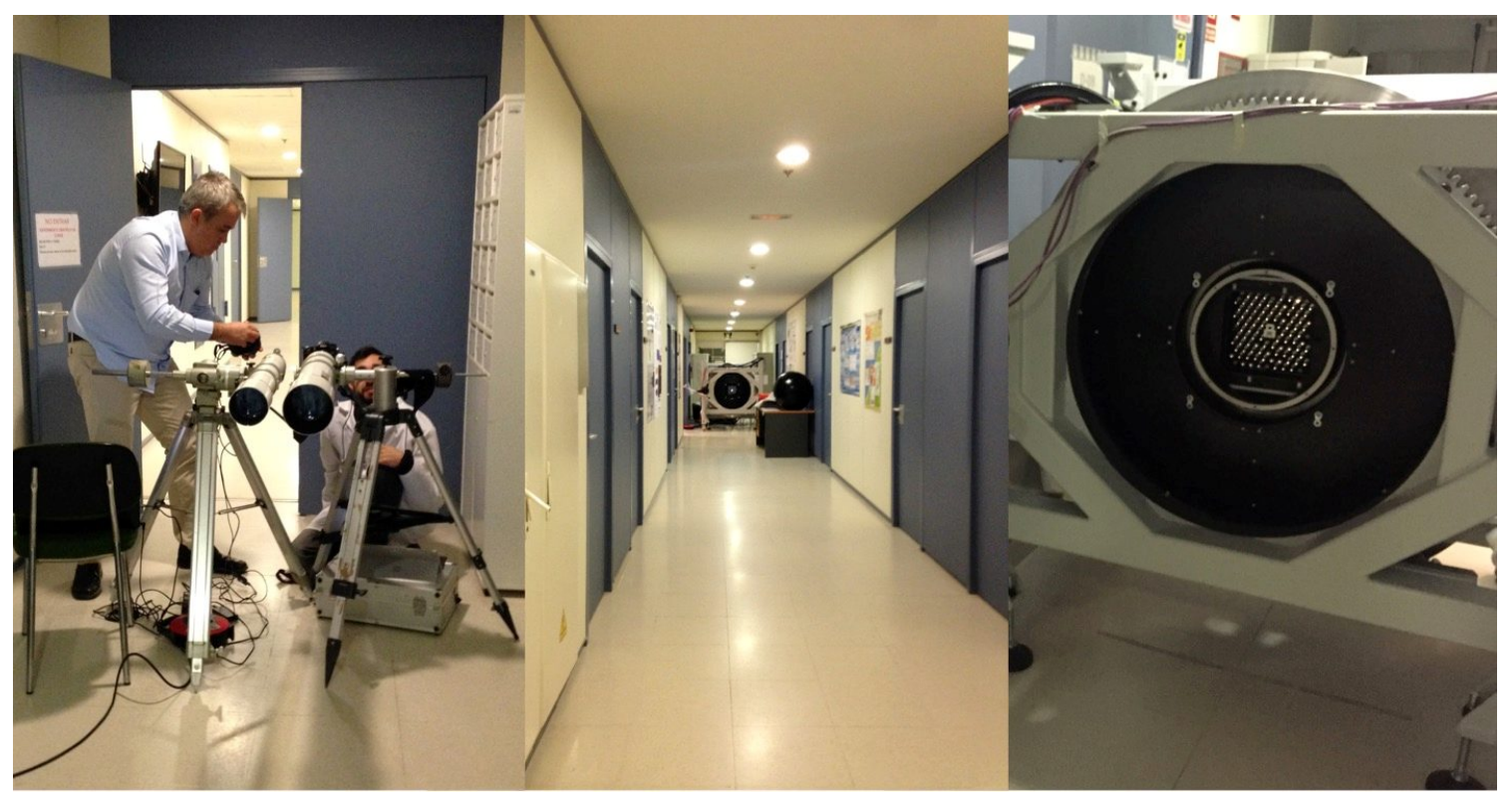

Figure 15: Positioners parameters laboratory verification set-up views. Left: Preparing telescopes, camera and CCD to register images. Center: Fiber MOS assembly at the opposite site of the corridor (18 m distance). Right: Fiber MOS minibundles back illuminated. 
Figure 15 shows the set-up for taking the MOS minibundles images. Two independent recording systems, including a Nikon D7100 (6000 x 4000 pixels, $3.89 \mu \mathrm{m}$ pixel) and a CCD QSI (2184 x 1472 pixels, $6.8 \mu \mathrm{m}$ pixel) both attached to $1000 \mathrm{~mm}$ focal length telescopes, were located at $18 \mathrm{~m}$ from the Fiber MOS focal frame, which is the position where the pupils converged.

Positioners were commanded at several positions in rotation 1 and rotation 2. Three images were taken at each position to allow average between them to reduce identification errors.

The centroids of the minibundles were identified by applying two different algorithms for the camera and the CCD. The result was a set of text files (one per image), which included pixels coordinates of the centroid of each minibundle. These coordinates were then analyzed for each minibundle to represent (see Figure 16) the trajectories followed by the positioners and to calculate from them the pointing parameters.
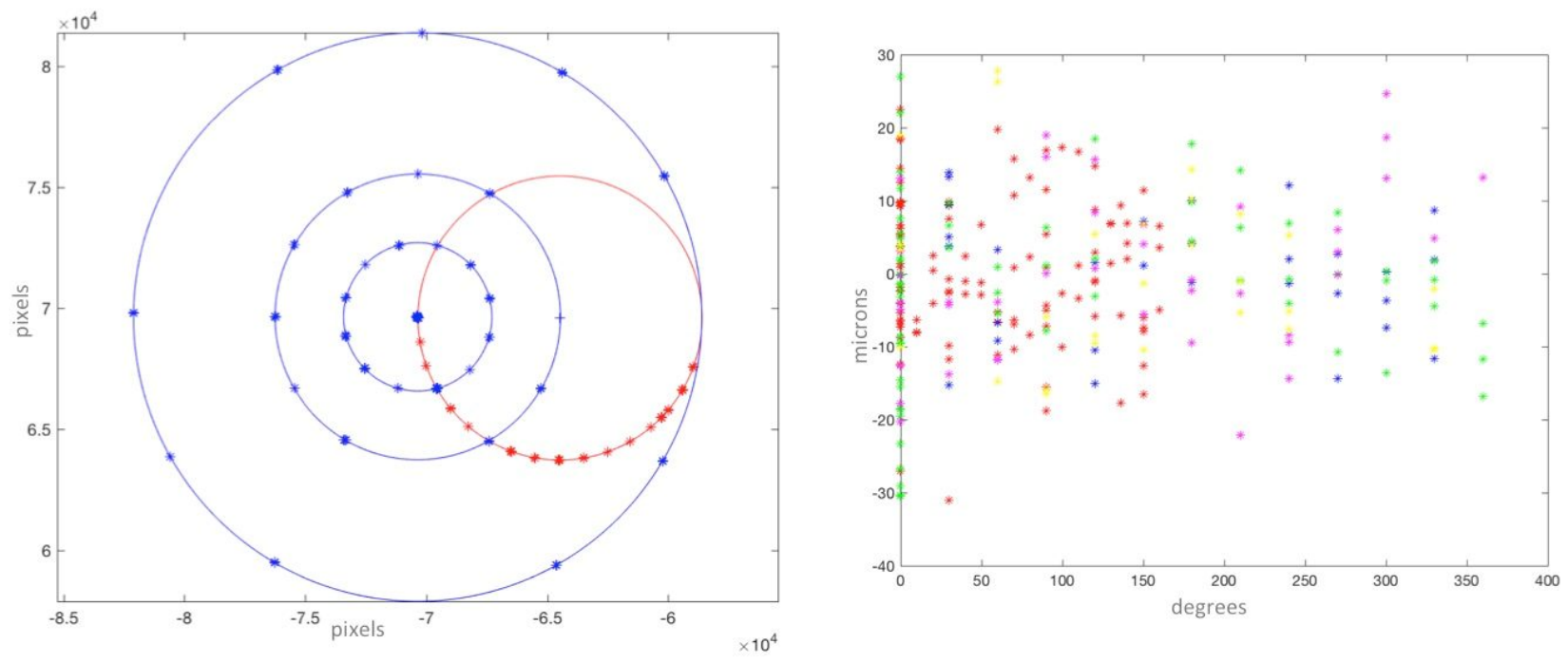

Figure 16: Left: Centroids positions in pixels when commanding. First, R1 from $0^{\circ}$ to $360^{\circ}$ in $30^{\circ}$ steps at R2 equals $0^{\circ}, 30^{\circ}, 60^{\circ}$ and $180^{\circ}$ and, second, R2 from 0 to $180^{\circ}$ in $10^{\circ}$ steps. Right: Errors in microns associated at each position. Both are for minibundle \#10.

The concordance between CCD and camera results and the measurements provided by AVS was in average $30 \mu \mathrm{m}$, which is adequate for achieving the pointing precision requirement.

\subsection{MOS pointing verification at GTC}

Prior to perform MOS pointing verification, the astrometric characterization of the MEGARA Folded-Cassegrain focal plane (FC-F) was performed with the aim to derive the accurate plate scale, the Instrument Alignment Angle (IAA), and the offsets between the reference (design) position of the instrument and the center of rotation of the FC-F. Once these parameters were known, GTC could maneuver with the required precision to acquire targets at the MOS minibundles and to perform the test to verify the positioning precision of the MOS mode.

\subsubsection{Astrometric characterization of the MEGARA Folded-Cassegrain focal plane}

The astrometric characterization of the FC-F focal plane was made during nighttime commissioning by carrying out a series of observations with the IFU and MOS that were combined in a petal-pattern mosaic composed of 6 MOS images around the nominal configuration to ensure that all the centroids where fully covered in all cases plus the corresponding LCB acquisition observation (see Figure 17). 
This test allowed to determine the plate scale, IAA angle and offsets between reference instrument center position and the rotator center and, also, showed that there is limited space for selecting a star for acquisition and guiding (A\&G) probes to change the guide star when offsetting from the position of the IFU to one of the MOS minibundles or in between two different minibundles. This implies that during few seconds (e.g.10s) to one minute the telescope would be tracking, which leads to uncertainties that are larger than our aimed precision for positioning of the robots. Due to this limitation, the determination of the MOS homing positions could not be carried out with individual measurements with each positioner but as part of the verification of the MOS pointing, which is described in the following section.

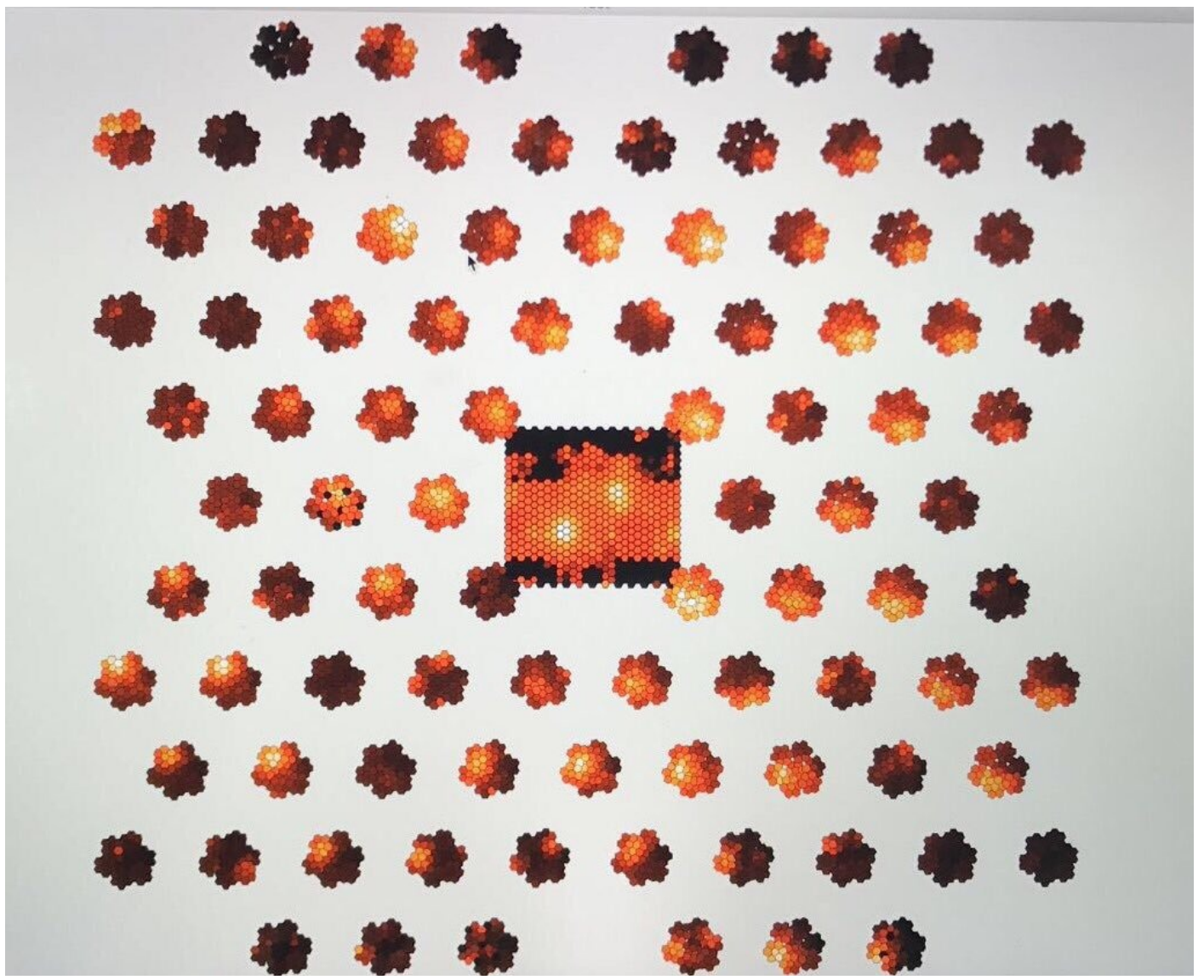

Figure 17: Reconstruction of the MOS petal-pattern mosaic observations of stars in Galactic globular cluster M71 along with the corresponding LCB acquisition observation. Note that although the orientations and sizes of each of the MOS minibundles are both correct, their central positions are not, as they have been moved close to the position of the IFU for displaying purposes. In total this image shows the result of 6 MOS observations and 1 LCB observation.

\subsubsection{Positioners pointing verification at GTC}

For the verification of the positioners pointing, we selected a target visible from GTC during a significant fraction of the night at the time of the second and third night-time commissioning runs (August 2017). Moreover, such a target should include enough bright stars to map the largest possible number of positioners with exposures of the order or below 15 minutes. The optimum choices were the globular clusters M15 [RA(J2000) = 21h29m58.33s; Dec(J2000) = 12 $\left.{ }^{\circ} 10^{\prime} 01.2^{\prime \prime}\right]$ and M71 [RA(J2000) $=19 \mathrm{~h} 53 \mathrm{~m} 46.49 \mathrm{~s} ;$ Dec $\left.(\mathrm{J} 2000)=18^{\circ} 46^{\prime} 45.1^{\prime \prime}\right]$. Finally, one MOS observation was performed with M71 and 6 different MOS configurations (plus the corresponding LCB acquisition observations; see below) were observed with the M15 cluster, which had the optimal visibility in the last nighttime commissioning runs.

The process to carry out the MOS observations was as follows. Once the target field was acquired with the GTC A\&G system to the cluster coordinates, an offset was commanded to the telescope to center the field to observe a reference star 
of the cluster in the center of the FOV in LCB/MOS FOV configuration. Then, the telescope starts guiding and an image was taken with the LCB mode in one MEGARA mode called LCB acquisition that allows to have an immediate feedback on real time of the position of the brightest target on the LCB. We compared the coordinates in which the reference star appears with the coordinates where it was supposed to be and applied the offset for correction; then we checked again the position of the reference star. After that, the offset was applied to make the star back to their nominal position in one of the positioners so that by applying this offset we assured the correct pointing and we commanded MOS positioners to the targets and take the MOS image with the MOS configuration. We took three images to be able to automatically correct the image from cosmic rays thanks to the on-line pipeline.

The observations were reduced with the MEGARA pipeline and displayed with MEGARA Quick Look Application (QLA) [4]. Then, the centroids errors in (x,y coordinates) were computed with respect to the center of each minibundle at the reduced images. Figure 18 shows the centroids errors for 4 different configurations.
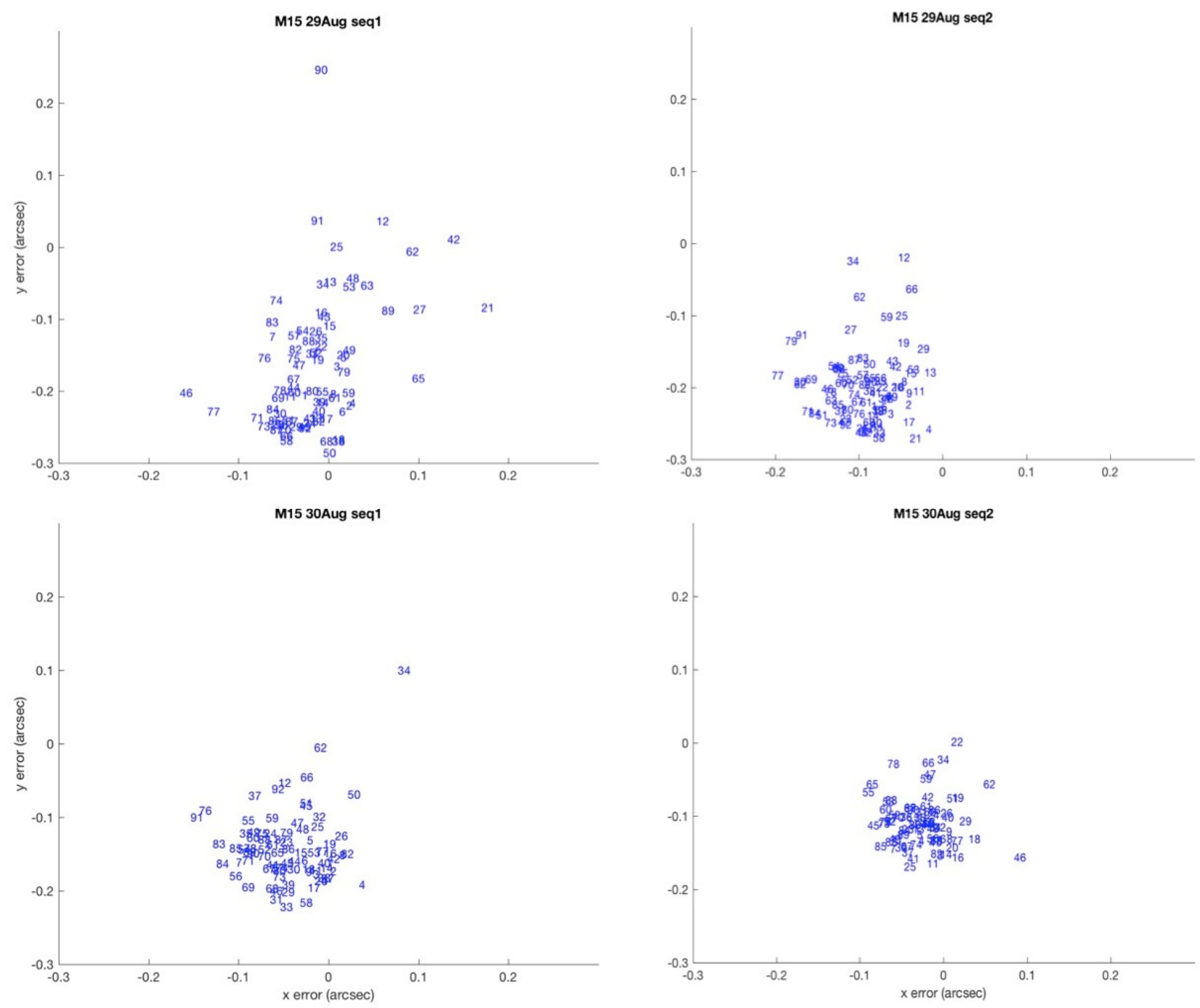

Figure 18: Pointing errors for 4 different observing configurations (positioners coordinates) for the cluster M15 carried out on August $29^{\text {th }}$ and August $30^{\text {th }} 2017$.

Table 3 summarizes the errors obtained from the 7 MOS measured configurations. Figure 19 shows the modules of the corrected errors vectors (ec) for all configurations and for each minibundle. 
Table 3: MOS observation pointing errors: em is the average of the error vector modules; (xc, $\mathbf{y c})$ are the average coordinates of the error vectors $(\mathrm{x}, \mathrm{y}), \mathbf{e c}$ is the average of the error vector modules after offsetting $(\mathbf{x c}, \mathbf{y c})$ coordinates from error vectors $(\mathrm{x}, \mathrm{y})$.

\begin{tabular}{|l|c|c|c|c|}
\hline Pointing configurations & em (arcsec) & xc (arcsec) & yc (arcsec) & ec (arcsec) \\
\hline M71 31 Jul & 0.113 & -0.009 & -0.005 & 0.113 \\
\hline M15 31 Jul & 0.135 & -0.080 & 0.022 & 0.114 \\
\hline M15 28 Aug seq 1 & 0.184 & -0.019 & -0.167 & 0.085 \\
\hline M15 28 Aug seq 2 & 0.226 & -0.098 & -0.198 & 0.055 \\
\hline M15 28 Aug seq 3 & 0.231 & -0.096 & -0.200 & 0.060 \\
\hline M15 30 Aug seq 1 & 0.159 & -0.051 & -0.142 & 0.054 \\
\hline M15 30 Aug seq 2 & 0.119 & -0.032 & -0.109 & 0.040 \\
\hline
\end{tabular}

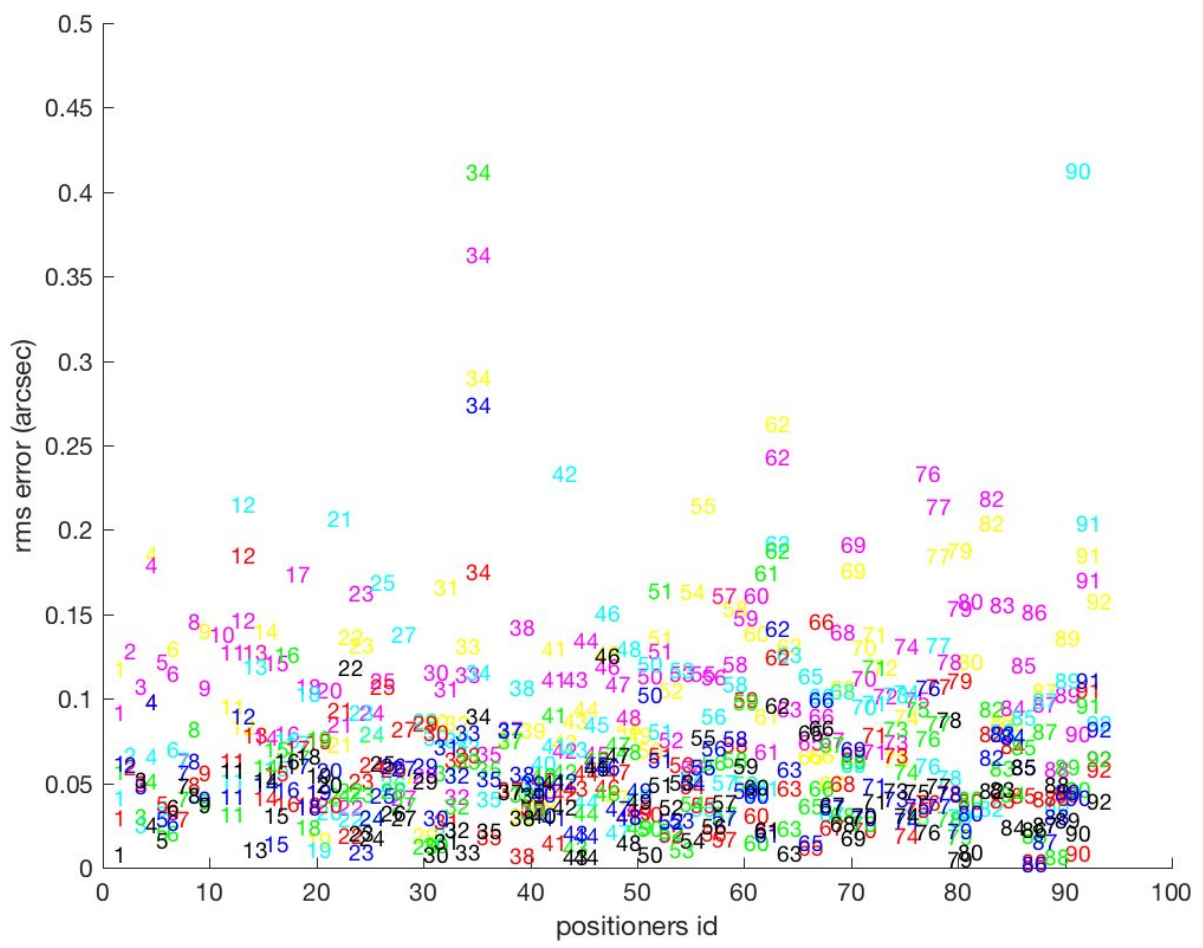

Figure 19: Summary of the pointing rms errors $(\operatorname{arcsec})$ for the 7 MOS configurations after offsetting correction (ec).

The first conclusion is that the center of the errors vectors has a displacement with respect to the reference position (taken from the LCB) in almost all images. We consider that this is an offsetting residual error that will be better corrected if during the acquisition process the final offset is calculated with a MOS fine acquisition (instead of with the LCB acquisition mode, as was done when taking these images).

The second conclusion is that, after applying the MOS fine acquisition correction, the average pointing error would be below $0.124 \operatorname{arcsec}(1 / 5$ of the spaxel size). 


\section{CONCLUSIONS}

This article describes the problems encountered and the solutions applied to fulfill the alignment and pointing requirements of the MEGARA MOS mode. We have described the error budget done to be able to assign manufacturing tolerances at each component. We have summarized the description and results of the tests carried out at both factory and laboratory, as well as the tools and set-ups developed for these tests. These early tests helped us to identify the problems and to find solutions and compensators. This paper has also described the different tests carried out at the GTC after delivery and integration during both daytime and nighttime commissioning periods.

The conclusion is that MEGARA MOS is fulfilling alignment and pointing requirements. The instrument was fully commissioning, it is operative and has been offered to the GTC community for the period 2018B to start observations in July 2018. The good design and a strict configuration control and test plan were crucial. However, this had not been possible without the hard work, imagination, and enthusiasm of the MEGARA team.

\section{REFERENCES}

[1] Gil-de-Paz, A. et al, " First scientific observations with MEGARA at GTC" Proc. SPIE 10702-43 (2018)

[2] Carrasco, E. et al, "MEGARA, the R=6000-20000 IFU and MOS GTC" Proc. SPIE 10702-42 (2018)

[3] Pérez-Calpena, A., et al., "Delivery and integration of MEGARA at GTC: the risky process of going from laboratory to the telescope", Proc. SPIE 10705-13 (2018)

[4] Gómez-Alvarez, P. et al, "MEGARA observation preparation and Quick Look software" Proc. SPIE 10707-56 (2018)

[5] Pérez-Calpena, A., et al., "MEGARA. High-precision alignment system for gluing fibers and microlenses", Proc. SPIE 9912-194 (2016)

[6] Pérez-Calpena, A., et al., "MEGARA Fiber bundles", Proc. SPIE 9147-211 (2014) 